\title{
Partial delamination detection and quantification in composite laminates using Laser Doppler
}

\section{Vibrometer}

\author{
Yashdeep Nimje
}

A Thesis Submitted to

Indian Institute of Technology Hyderabad

In Partial Fulfillment of the Requirements for

The Degree of Master of Technology

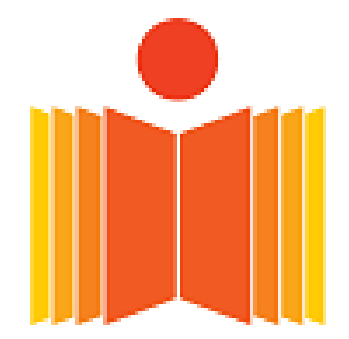

भारणीय प्रौचोगिकी चैस्थान एै:्राबाद

Indian lestitute of Technslogy Hyderabed

Department of Mechanical Engineering

june 2016 


\title{
Declaration
}

I declare that this written submission represents my ideas in my own words, and where ideas and words of others have been included, I have adequately cited and referenced the original sources. I also declare that I have adhered to all principles of academic honesty and integrity and have not misinterpreted or fabricated or falsified any idea/data/fact/source in my submission. I understand that any violation of the above will be a cause for disciplinary action by the Institute and can also evoke penal action from the sources that have thus not been properly cited, or from whom proper permission has not been taken when needed.

\author{
Qinge \\ (Signature) \\ Jashdeep. P. Ninge \\ (Student Name) \\ me14mtech 11038
}

(Roll No.) 


\section{Approval Sheet}

This thesis entitled "Partial delamination detection and quantification in composite laminates using Laser Doppler Vibrometer" by Yashdeep Nimje is approved for the degree of Master of Technology from Indian Institute of Technology (IIT) Hyderabad.

$$
M \cdot \dddot{m}
$$

(Dr. Ramji M, Assoc. professor) Examiner Dept. of Mechanical and Aerospace Engineering

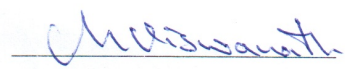

(Dr. Viswanath Chinthapenta, Asst. professor) Examiner Dept. of Mechanical and Aerospace Engineering

$$
\text { snichl }
$$

(Dr. Syed Nizamuddin Khaderi, Asst. professor) Examiner

Dept. of Mechanical and Aerospace Engineering

$$
\text { R. Yat. Than }
$$

(Dr. Gangadharan Raju, Asst. professor) Adviser

Dept. of Mechanical and Aerospace Engineering

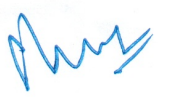

(Dr. S. Suriya Prakash, Assoc. professor) Chairman Dept. of Civil Engineering 


\section{Acknowledgements}

I would like to thanks my adviser Dr. Gangadharan Raju for giving me such opportunity to work in this area, also for giving me continuous motivation throughout my work. I also wish to thank him for his guidance and sharing his vision of the Structural Health Monitoring concepts and provided many ideas at all stages of this research effort. Exchange of ideas with Dr. Gangadharan Raju throughout the research effort were crucial to the successes reported in this thesis. I want to express my thanks to my IITH friends and lab assistant for their constant support and encouragement throughout this work. I would like to thanks Dr. Ashok Kumar Pandey sir for giving me permission to use the Vehicle Dynamics Lab and special thanks to Mr. Prasant kambli for teaching me LDV equipment. I want to express my thanks to workshop members, especially Mr. A.Pravin, Mr. Pramod for helping me in fabrication of composite specimens throughout this research work. 


\section{Dedication}

To my family and teachers 


\begin{abstract}
Vibration based structural health monitoring techniques are widely used for detection and quantification of delamination in composite structures. In this work, non-contact vibration measurement technique namely Laser Doppler Vibrometer (LDV) is used for damage characterization of partial delamination in composite beams. The modal information such as natural frequencies and mode shapes of composite beam are obtained using LDV and validated with finite element models for comparison with the obtained experimental results. Mode shape based damage detection algorithms namely, fractal dimension approach is used for quantification of delamination in composite beams. In the present work, a modified generalized fractal dimension technique is proposed to locate delamination of different sizes and shapes in the composite beam. Different types of partial delamination like C-type, I-type in composite beam specimens were fabricated and experimentally studied using LDV. The modified fractal dimension technique was then applied to the mode shapes results of the various partial delamination to quantify the damage. In addition, multiple delaminations were introduced in composite plates and experiments were carried out using LDV. Modified fractal dimension technique was then applied to quantify the delamination in composite laminates.
\end{abstract}




\section{Contents}

Declaration ...................................... ii

Approval Sheet . . . . . . . . . . . . . . . . . . . iii

Acknowledgements ......................... . . iv

Abstract .......................... . . . . . . . . .

$\begin{array}{ll}\text { Nomenclature } & \text { viii }\end{array}$

1 Structural Health Monitoring $\quad 1$

1.1 Introduction . . . . . . . . . . . . . . . . . . . . . 1

1.2 Strain based SHM . . . . . . . . . . . . . . . . . . . . . . 1

1.3 Impedance based SHM . . . . . . . . . . . . . . . . . . . . . . . . . 2

1.4 Ultrasonic wave based $\mathrm{SHM} \ldots \ldots \ldots$. . . . . . . . . . . . . . . 2

1.5 Vibration based SHM . . . . . . . . . . . . . . . . . . . 2

1.6 Damage detection in composite structures . . . . . . . . . . . . . . . . . 3

1.7 Literature survey . . . . . . . . . . . . . . . . . . . . . . . . 4

1.8 Scope and Objective . . . . . . . . . . . . . . . . . . 5

1.9 Thesis Layout . . . . . . . . . . . . . . . . . . . . . . . . 5

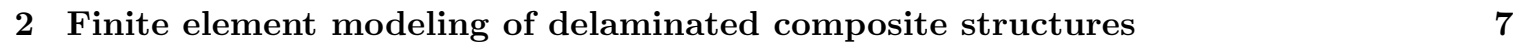

2.1 Introduction . . . . . . . . . . . . . . . . . . . . . 7

2.2 Literature review . . . . . . . . . . . . . . . . . . . . 7

2.3 Finite element modeling . . . . . . . . . . . . . . . . . . . 8

2.3.1 Modeling of delamination . . . . . . . . . . . . . . . 8

2.4 Numerical validation - different types of delamination . . . . . . . . . . . . . . 9

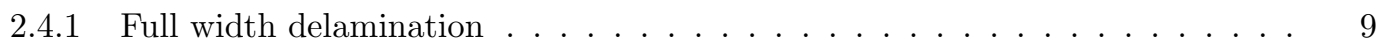

2.4 .2 Partial widthwise delamination . . . . . . . . . . . . . . . 11

2.4 .3 Multiple delamination ....................... 12

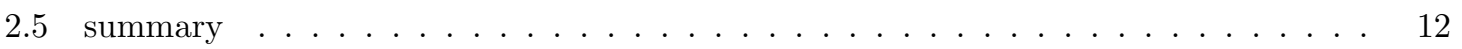

3 Vibration analysis of composite structure $\quad 14$

3.1 Introduction . . . . . . . . . . . . . . . . . . . . . . 14

3.1.1 Theory and specification of LDV . . . . . . . . . . . . . . . 14

3.2 Principle of Doppler Effect . . . . . . . . . . . . . . . . . . . . . . . . . 14

3.3 Experimental modal mnalysis using LDV . . . . . . . . . . . . . . . . . . . . . 16

3.3.1 Specimen Fabrication . . . . . . . . . . . . . . . 17 
3.4 Material characterization of composite structure . . . . . . . . . . . . . . . 18

3.4.1 Governing differential equation of plate . . . . . . . . . . . 18

3.4.2 Rules for the selection of the weighting function . . . . . . . . . . . . 18

3.4.3 Selection of mode shape . . . . . . . . . . . . . . . . . . . 20

3.5 The Regressive fourier series . . . . . . . . . . . . . . . . . . . . . . . 20

3.5.1 Experimental results for bending stiffness of the composite plate . . . . . . 23

3.6 Summary . . . . . . . . . . . . . . . . . . . . . . . 24

4 Damage characterization approaches $\quad 25$

4.1 Introduction . . . . . . . . . . . . . . . . . . . . . 25

4.2 Literature review . . . . . . . . . . . . . . . . . . . . 25

4.3 Frequency based method . . . . . . . . . . . . . . . . . . . . 26

4.4 Mode shape-based method . . . . . . . . . . . . . . . . . . . . 26

4.4.1 Traditional mode shape change method . . . . . . . . . . . . . . 27

4.4 Curvature based method . . . . . . . . . . . . . . . . 28

4.4.3 Generalized fractal dimension technique . . . . . . . . . . . . 30

4.5 Summary . . . . . . . . . . . . . . . . . . . . . . 37

5 Conclusion and Recommendation for future work 38

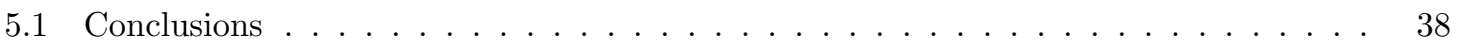

5.1 .1 Numerical work . . . . . . . . . . . . . . . . . . . 38

5.1 .2 Experimental work . . . . . . . . . . . . . . . . . 38

5.2 Future work . . . . . . . . . . . . . . . . . . . . 39

$\begin{array}{lr}\text { References } & 40\end{array}$ 


\section{Chapter 1}

\section{Structural Health Monitoring}

\subsection{Introduction}

Over the last two decades, there is a growing need for real time monitoring of structures made of composite materials to avoid catastrophic failures and provide confidence for the usage of these high performance materials into aerospace, marine and wind energy sectors. Unlike metals, failure mechanism are quite complex in composites involving fiber breakage, fiber pull-out, matrix cracks, fiber-matrix de-bonding and delamination. An important limitation of most of the existing non destructive evaluation (NDE) methods is that they are off-line, that is, the normal operation of the structure has to be interrupted and the device often has to be disassembled for inspection. In addition the conventional NDE equipments are bulky, consume more power, less cost effective and cannot be easily integrated into the structure which makes them not suitable for real time monitoring. The above requirements has led to the recent advances in sensor technology, electronic packaging, manufacturing and signal processing algorithms which resulted in modification or adaptation of the conventional NDE techniques suitable for real time continuous monitoring and automated supervision of the overall health of structures. This technology of structural integrity evaluation is known as Structural health monitoring and it is expanding rapidly with new technologies and lots of potential applications in aerospace, civil and mechanical structures. A complete SHM approach consists of four basic steps (1) identification of damage occurrence in the structure, if any, (2) identification of damage locations, (3) quantification of damage, and (4) evaluation of structural performance and its residual life. Many SHM techniques thus have been developed to quantify and locate the damages in the structures, based on either the global or the local interrogation of the structures. SHM can be carried out by looking at natural frequency of the structures, electromechanical impedance based measurement, the strain based measurements and amplitude or energy of the ultrasonic guided waves reflected from edges and defects in the structure.

\subsection{Strain based SHM}

Very few works has been reported in literature on the development of strain based SHM compared to other methodologies [1]. Strain gauges are widely used in SHM systems because they are inexpensive, easy to install, and sensitive enough to detect the potential danger of a structure. However, off- 
the-shelf equipment for the strain gauges is usually either wired to the sensors or wireless with very limited range, which significantly increases the total cost as well as restricting the performance of the deployment [2]. Katsikeros [3] proposed an innovative strain based SHM methodology, which were processed by a back-propagation feed-forward artificial neural network (ANN) to detect damage in an aircraft lap joint structure. The potential drawback of strain based SHM is the requirement of a high number of strain measurements in the vicinity of the damaged area which can be efficiently compensated by the utilization of bragg grating optical fibers as strain sensors [4].

\subsection{Impedance based SHM}

Impedance-based SHM has been implemented on laboratory structures as well as a few in situ applications $[5,6,7]$. The principle of the impedance approach is based on the transduction mechanism of the sensor bonded to the structure which uses high frequency vibrations to monitor the changes in structural impedance that would indicate damage or imminent damage. A commonly used transducer material for this approach is a piezoelectric ceramic (Lead zirconate titanate (PZT)) which can be readily bonded and suited to the desired application. By exciting the PZT and monitoring the current drawn, the impedance of the transducer is determined. This impedance would change depending if the structure is damaged, as the stiffness of the structure would change.Advantages of impedance-based SHM are being insensitive to changes in boundary conditions or operational vibrations, their model-independent nature makes them applicable to complex structure and low power requirements for excitation of the PZT. Though impedance based methods are sensitive to small amounts of damage, their sensitivity drops off dramatically with increasing distance between the damaged location and the transducer.

\subsection{Ultrasonic wave based SHM}

On the other hand, the local or wave based approach is based on the interaction of propagating elastic waves with the structure. Elastic wave propagation in the structure can be classified into bulk waves, Lamb waves, Rayleigh or surface waves, Love waves, Stonley waves etc. The idea behind this approach is when the elastic wave encounters any damage, the following things can happen a) waves get either reflected or scattered depending upon the defect characteristics b) waves undergo dispersion or attenuation across the damage resulting in reduction in amplitude and time spread of the wave c) mode conversion of the wave and d) generation of higher harmonics. The wave based approach uses one of the above wave parameters for detection of damage in the structure. Aranguren et al. [8] represents Phased Array Monitoring for Enhanced life Assessment. Piezoelectric sensors and actuators are integrated and is able to generate excitation signals. The generated signal are sent through piezoelectric actuators, acquire the received signals in the piezoelectric sensors, and carry out signal processing to check the health of structures.

\subsection{Vibration based SHM}

Vibration based Structural Health Monitoring (SHM) is one of the major approaches for inspection and structural integrity evaluation.Doebling [9] introduce the various method based on vibration. 
The global or vibration based approach looks for the change in the modal characteristics of the structure for the occurrence of damage. The principle behind this approach is that damage alters the stiffness, mass or dynamical properties of the structure, which in turn affects the modal response of the structure. Reliable modal based methods are essential to detect and quantify damages for implementation of robust SHM systems. The challenges are to identify the changes in the physical parameters due to damage in the specimen and correlate them with the corresponding measured modal parameters. Scanning Laser Doppler Vibrometer(LDVs) are commonly used in the vibration community to measure the velocity of vibrating surface over a broad area of interest. In this technique, the laser light reflected back to sensor from the structure is compared to the reference beam and relative shift in frequency between reference beam and measured beam will correlate to the surface velocity. As opposed to the other optical techniques LDV technique is easy to obtain transfer function. i.e frequency response function(FRF). As change in FRF gives information of healthy and damage structure. Since it is non-contact and non-mass loading technique vibration measurement system. Y.Zou et al [10] gave the brief review on the vibration based delamination detection technique.

\subsection{Damage detection in composite structures}

Among the various failure modes, delamination is a barely visible damage induced by transverse impact, and results in significant reduction of the strength and stiffness of the composite. Delamination is a damage type that generally occurs by transverse cracking in individual plies as shown in Fig. 1.1. This damage is due to de-bonding happening between individual plies of a laminate. The crack grows in a plane parallel to the fibers, but at the interface between two layers. Although delamination do not lead to complete fracture, but they can seriously affect the load carrying capacity of a laminate. Delamination can alter the dynamic responses such as natural frequencies, mode shapes and these changes can be extracted to estimate damages in the structure by modal analysis [11]. The present research work focuses on developing the relationship between the dynamic

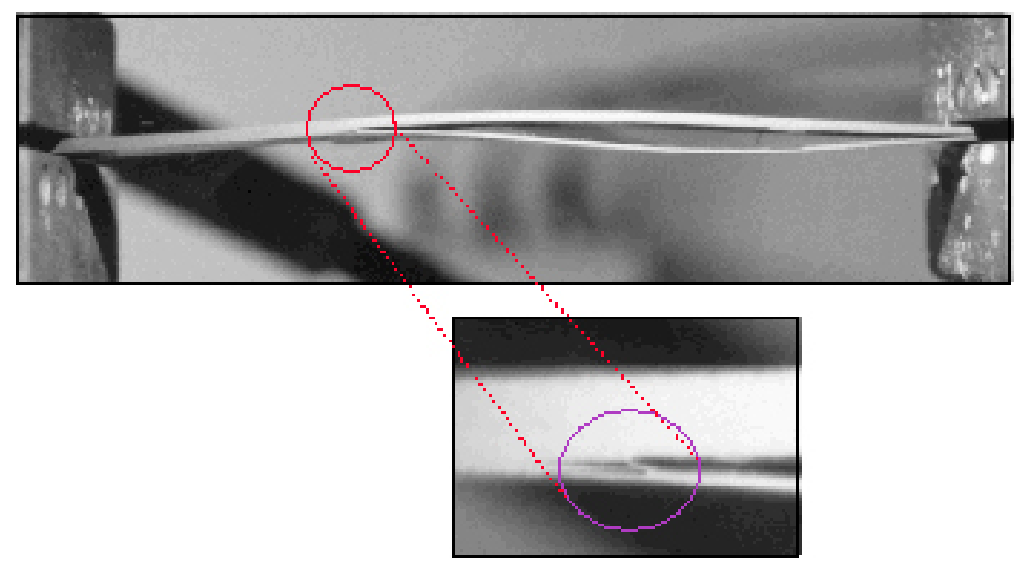

Figure 1.1: Internal Delamination in composite

response of healthy and the damage composite beam as a basis of the damage identification and quantification for composite structures. 


\subsection{Literature survey}

Vibration-based SHM provides an identification method with basic idea that the modal parameters such as natural frequencies, mode shapes, and frequency response functions (FRFs) are functions of the physical properties of the structures such as stiffness, mass, and modal damping. Therefore, the changes in these dynamic parameters can be used to locate and assess damages. As the dynamic behavior of a structure is a function of these physical properties and will therefore directly be affected by the damage. The dynamic behavior can be described by time, frequency and modal domain parameters. These three SHM parameters gives information of presence of damage. Various modal parameters like natural frequency, mode shape and modal curvature are affected by the delamination present in the composite structure and their sensitivity depends on different aspects, such as the size and the location of the damage Ramkumar et al. [12] proposed a beam model to study the effect of delamination on the natural frequency of a delaminated beam. They modeled the beam with a through-width delamination by using four timoshenko beams. They neglected the effect between the axial and transverse vibration. Wang et al. [13] improved the model of ramkumar et al. [12] for an isotropic beam by including the effect of coupling between axial and transverse vibration. Mujumdar and suryanarayan [14] used four beam elements to model through width delamination of composite beam. They used to beam elements above and below the delamination and constraints were used to join them to the adjacent healthy beam elements. Chakraborty [15] used the first 10 modal frequencies generated from finite element model (FEM) of a delaminated composite plate to train a back neural network to predict the size, shape and location of damage. The graphical technique was first proposed by adams and cawley [16], who showed that by plotting a crack size index vs. crack location for the first three modes of a straight bar, the possible size and location can be estimated. Della and Shu* [17] proposed various analytical models and numerical analysis for free vibration study of delaminated composite beams, they also represent delamination on the frequencies and mode shapes of composite laminates Nagesh Babu and Hanagud [18] developed a one dimensional finite element (FE) model for composite beams by using the classical laminate theory (CLT). Krawczuk et al [19] and Zak et al. [21] used the free mode assumption in their studies. They used beam finite element three nodes three degree of freedom per node. Ju et al. [22] presented a model for composite beam with multiple delaminations Three noded, three degrees of freedom isoparametric beam elements were used to discrete the delaminated beam. Campanelli and engblom [23] develope and FE model for laminated composite plates. The model uses 8 noded shear deformable plate elements. Krawczuk et al. [20] and zak et al. [21] used 8 noded plate elements with five degrees of freedom per node. The continuity of transverse displacement, axial displacements and rotation were satisfied at the delamination junctions. Okafor et al. [24] used first four normalized frequencies of the composite beam to train a neural network and then applied to predict delamination size from the measured frequencies in delaminated composite beam. Valoor and Chandrashekhara [25] trained a back propagation neural network to predict both delamination size and location by using the natural frequencies obtained from a thick beam model which incorporated shear deformation and Poisson effect. Zhifang Zhang et al. [26] presented an inverse algorithm for solving the non linear equation to predict the interface, lengthwise location and size of delamination. This algorithm have been validated using numerical data generated from the finite element model (FEM) of delaminated beams and measured frequencies from modal testing conducted on simply supported and cantilever plates. Shen et al. [27] experimentally studied the vibration behavior 
of 8-layer $\left([0 / 90 / 0 / 90]_{s}\right)$ carbon-epoxy beams with delaminations of four sizes $(25.4,50.8,76.2$ and $101.6 \mathrm{~mm}$ ) centrally located at four interfaces Kumar et al. [30] developed finite element models to study various type of partial delamination and heir effects on the modal parameters.Lestari et al. [35] proposed a combined analytical and experimental modal approach to locate delamination in composite beams using curvature information of the mode shapes. They used surface bonded piezoelectric wafers to capture the mode shape of composite structures and evaluated the curvature to locate the delamination. Farrar and Doebling [9] present work to assess the vibration response of the structure in order to detect, localize and quantify the damage. This method have used the difference in natural frequencies and mode shape between undamaged and damage structure due to modification of the structural. Kessler et al [32] studied about damage detection method using the frequency approach Helfrick et al. [33] applied 3D digital image correlation(DIC) methods to measure shape and deformation of a vibrating structure for characterization of damage. .WeiFan and Pizhong Qiao. [34] proposed generalized fractal dimension technique to quantify the effect of delamination on the composite beam. They modified the fractal dimension technique which was introduced by Hadjileontiadisa [35] in 2005. Qiao et al. [34] used laser Doppler vibrometer (LDV) for damage detection in composite plates using modal curvature and developed algorithms based on global smoothing method and fractal dimension technique to locate the delamination. From the above literature survey, not many works have been reported on the study of partial and multiple delamination in composite structures using vibration based SHM and this problem is studied in detail using numerical and experimental approaches.

\subsection{Scope and Objective}

Composite structures are more prone to delamination kind of failure due to bird/hail impact, tool drops etc. and this significantly reduces the strength of the structure. The objective of this work is to identify the presence of delamination in composites, locate and quantify the dimension or size of the delamination. In this work, we are planning to investigate full width delamination, partial delamination like C-type, I-type and multiple delamination in GFRP composite specimens. The scope of the work is given by 1) Finite element modeling of various kinds of delamination and studying their effect on the modal parameters like natural frequencies, mode shapes and their curvature. 2) To study the mode shapes of the delaminated beam using various signal processing methods to detect, locate and quantify the size of the delamination. 3) Experiments are carried out using LDV on various kind of delaminated specimens to obtain the natural frequencies and mode shape 4) Signal processing methods are used to study the experimentally obtained mode shapes for delamination characterization.

\subsection{Thesis Layout}

In second chapter the finite element modeling of the delaminated composite structure is discussed in detail. In third chapter, we discuss the basic principles behind laser Doppler vibrometer, details of the experimental setup and the procedure followed on the LDV were presented. In fourth chapter, damage detection technique based on frequency, mode shape, and the curvature of the specimen were discussed in detail and their application to quantify the damage. In the final chapter, the 
conclusions from the present work and recommendation of the future work is presented. 


\section{Chapter 2}

\section{Finite element modeling of delaminated composite structures}

\section{$2.1 \quad$ Introduction}

Laminated composite structures are anisotropic and heterogeneous in nature and their failures are quite complex. Finite element modeling approaches are widely used for simulation of various damage mechanism like fiber failure, matrix cracking and delamination etc. In this work, finite element framework is used for modeling the delamination and modal analysis is further performed to investigate their effects on the natural frequency and mode shapes.

\section{$2.2 \quad$ Literature review}

Among the numerical tools, finite element method is widely used for modeling composite laminates and different elements like 1D beam, 2D shell/plate, continuum shell and 3D brick elements are used to capture the modal behavior of composites. Ramkumar et al. [12] used Timoshenko beam elements to model through width delamination and studied their modal characteristics. Majumdar and Suryanarayanan [14] used Euler beam elements and developed a constraint model to simulate delamination at arbitrary location in lengthwise and thickness directions. Kumar et al. [30] proposed a beam finite element model capable of handling multiple partial width delaminations. Delamination in plates can be modeled using two approaches, namely, region wise and layer wise approach. In region-wise approach, the delamination region is divided into sub laminates and later displacement/force continuity conditions are introduced between healthy and delaminated regions. In layer-wise approach, delamination is introduced by defining additional nodes along the interface and not enforcing any displacement/force continuity along the interface function each divided into to study Zhang et al. [26] proposed an inverse algorithm equation to predict the location of the delamination in the lengthwise and thickness direction and predicted the size of delamination.They used 3D brick elements to model the simply supported and cantilever CFRP delaminated specimens and performed modal analysis to validate their algorithm for quantifying the damage .Kumar et al [30] developed the Finite element analysis on various type of delamination by using COMSOl and validate his results with standard literature of of shen and grady [27].A shell element gives the 
accurate result for the healthy specimen but the only drawback is that contact elements are not able to hold the intact surface, it splits into two parts while performing the processing. So we used 3D solid brick elements to model the specimen, drawback related with the 3D solid elements is that it makes the specimen more stiff when ratio of thickness to width decreases. In this work, layer wise approach is followed to simulate the partial delamination along the lengthwise and widthwise direction of the laminate. Additional nodes are introduced along the delamination interface and no constraints are enforced to simulate the delamination. Further to avoid inter penetration of the delaminated sub-laminates, contact elements are introduced along the interface.

\subsection{Finite element modeling}

In this work, finite element model is generated for composite laminated beams to determine modal parameters of glass fiber reinforced plastic (GFRP) beams with delaminations. Modeling was done using standard FEA package ANSYS 14. For our numerical study, unidirectional laminate $[0]_{4}$ is considered. For modeling the composite laminate, a 3D SOLID185 8 noded brick element was used. The mesh size of the element is governed by the thickness of each lamina and the mesh density is very high for thin laminates. This increases the computational effort and time. In addition, shear locking problem of brick element is encountered when modeling thin laminates which can be resolved by using reduced integration. Despite these limitations, the brick element can capture the mode shapes accurately which is required for characterizing the delamination in the composite.

\subsubsection{Modeling of delamination}

The delamination in the laminate is modeled using a layer-wise approach. Along the delamination interface, additional set of nodes were introduced. Subsequently constraints were introduced along the interface by using contact FE elements. The nodes associated along the interface of un-delaminated area are merged together with the help of contact elements (target170/contact173) and choosing 'bonded always' option in the contact manager. While the nodes in delaminated interface were kept separated and contact elements used to avoid the penetration of sub-laminates of delaminated beam. Target 170/ Contact 173 are four node 2D elements available in Ansys. Usage of the contact elements in the delaminated region makes the problem non-linear and can capture the motion of the delamination interfaces more accurately. In our work, the focus is on capturing the linear modal behavior characteristics of laminates and linear eigen solver was chosen to perform the modal analysis. The nonlinear part of delamination interaction is ignored in this work. A convergence study was carried out to determine the optimal mesh density required for modal analysis of delaminated composite laminates. For this study, a laminate specimen of having stacking sequence of $[0 / 90]_{2 s}$ with length of the specimen $=0.127 \mathrm{~m}$, width $=0.0127 \mathrm{~m}$ and ply thickness $=0.000127 \mathrm{~m}$ was considered.The material elastic properties are given by $E_{11}=134 \mathrm{GPa}, E_{22}=10.3 \mathrm{GPa}, G_{12}=5$ $\mathrm{GPa}, \gamma_{12}=0.33$, Density $=1480 \mathrm{~kg} / \mathrm{m} 3$. From the convergence study results, an optimal mesh size of 100 elements in the lengthwise direction, 20 elements in the widthwise direction and one element for each lamina across the thickness of the beam was chosen. To extract the modal parameters block lancoz method was chosen and the five modes were extracted for the numerical study. 


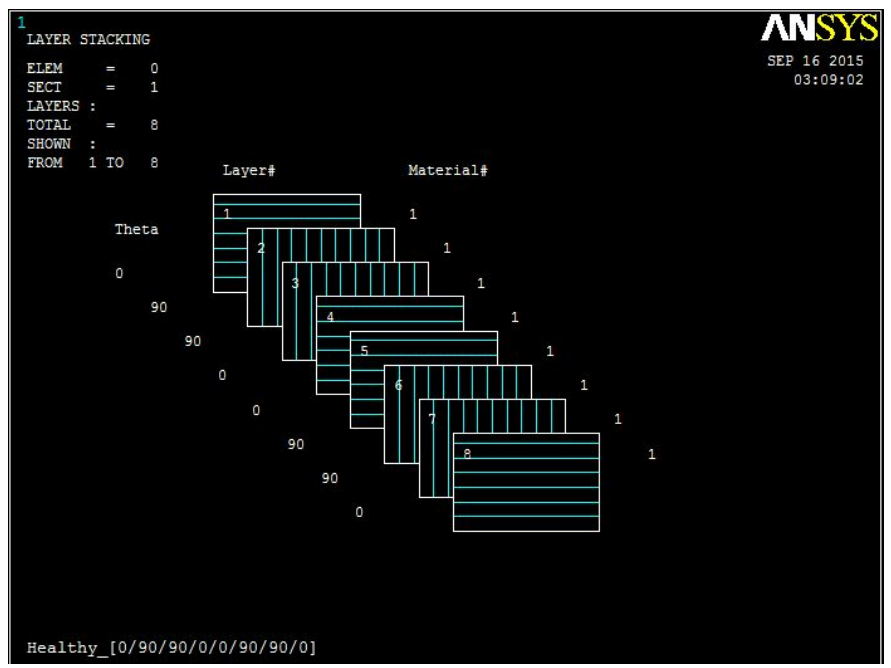

Figure 2.1: 8 layer $[0 / 90]_{2 s}$ FEA model

\subsection{Numerical validation - different types of delamination}

In this section, different kinds of delamination were modeled using Ansys and modal analysis is performed to extract the natural frequencies and mode shapes of the laminate.

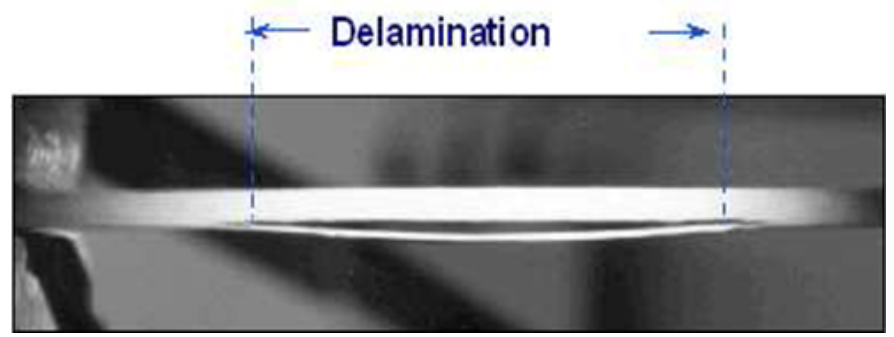

Figure 2.2: Specimen with delamination

\subsubsection{Full width delamination}

A cantilever beam made up of GFRP composite with the stacking sequence $[0 / 90]_{2 s}, 0.127 \mathrm{~m}$ length, $0.0127 \mathrm{~m}$ breadth and $1.016 \mathrm{~mm}$ thickness is considered The material properties of the beam is $E_{11}=$ $134 \mathrm{GPa}, E_{22}=10.3 \mathrm{GPa}, G_{12}=5 \mathrm{GPa}, \gamma_{12}=0.33$, density $=1480 \mathrm{~kg} / \mathrm{m} 3$. The numerical simulation perform in the literature.[29] at different layer with the help of the COMSOL. The different stacking sequence is shown in this fig. 2.3. Fig. 2.4 indicates the schematic diagram of a delaminated composite beam. The natural frequency results of the delaminated laminate obtained from ANSYS modeling are shown in table 2.1. The FE simulation results from the in table 2.1 closely matches the literature results [29]. Furthermore, the natural frequency results when the delamination region are present in third and fourth layer are shown in table 2.2 and table 2.3 respectively. 


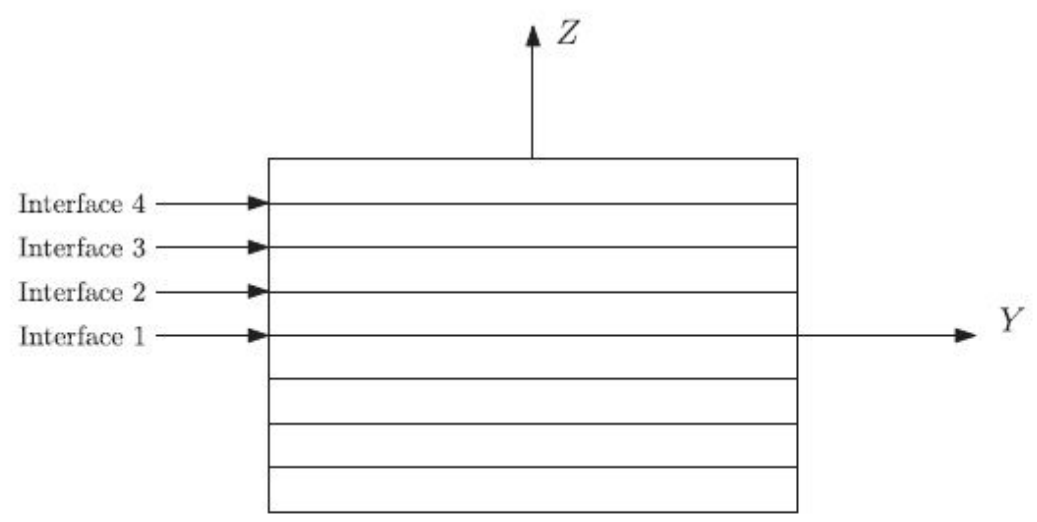

Figure 2.3: Different layer of composite

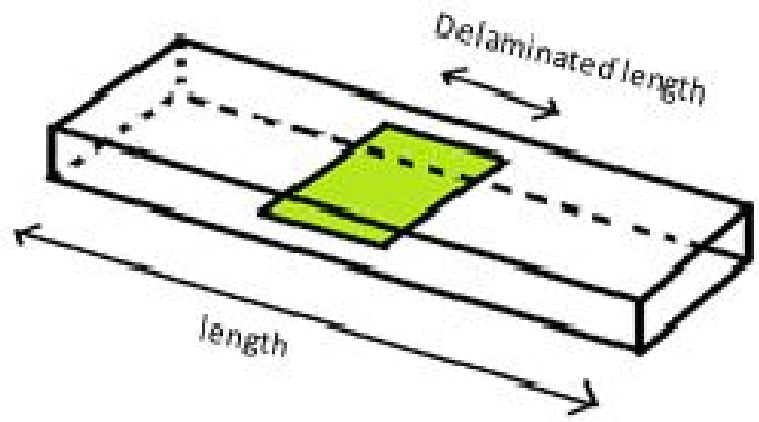

Figure 2.4: Full widthd elamination

\begin{tabular}{|c|c|c|}
\hline delamination in lengthwise $(\mathrm{mm})$ & S.Kumar $(\mathrm{Hz})$ & FEA results $(\mathrm{Hz})$ \\
\hline 0 & 81.46 & 81.4 \\
25.4 & 81.43 & 80.255 \\
50.8 & 76.04 & 74.634 \\
76.2 & 67.73 & 64.69 \\
101.6 & 53.65 & 53.9 \\
\hline
\end{tabular}

Table 2.1: 1st layer delamination

\begin{tabular}{|c|c|c|}
\hline delamination in lengthwise $(\mathrm{mm})$ & S.Kumar $(\mathrm{Hz})$ & FEA results $(\mathrm{Hz})$ \\
\hline 0 & 81.46 & 81.4 \\
25.4 & 81.39 & 80.356 \\
50.8 & 79.11 & 75.145 \\
76.2 & 67.93 & 65.711 \\
101.6 & 58.69 & 55.183 \\
\hline
\end{tabular}

Table 2.2: 2nd layer delamination 


\begin{tabular}{|c|c|c|}
\hline delamination in lengthwise $(\mathrm{mm})$ & S.Kumar $(\mathrm{Hz})$ & FEA results $(\mathrm{Hz})$ \\
\hline 0 & 81.46 & 81.4 \\
25.4 & 81.76 & 81.106 \\
50.8 & 78.58 & 79.501 \\
76.2 & 75.15 & 79.04 \\
101.6 & 62.82 & 64.642 \\
\hline
\end{tabular}

Table 2.3: 3rd layer delamination

\subsubsection{Partial widthwise delamination}

The schematic diagram of the partial delamination, namely, C-type and I-type is shown in Fig. 2.5 Colored portion in the diagram indicates the presence of the delamination, it is generally made

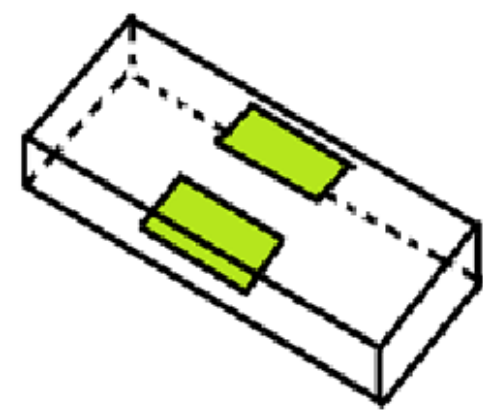

a)

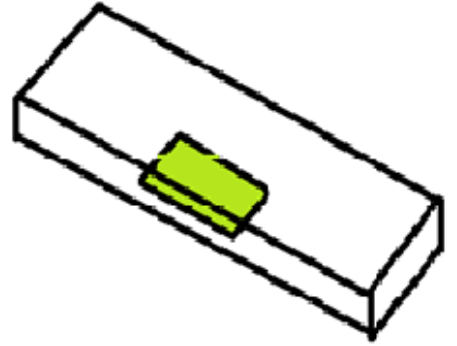

b)

Figure 2.5: a)I-type delamination. b)C-type delamination

by inserting the teflon tape of $0.09 \mathrm{~mm}$ between the layer. The C-type delamination of dimensions $50.8 \mathrm{~mm}$ in lengthwise direction and width is varied in terms of percentage of actual width of the laminate. The modal analysis results of the C-type and I-type are presented in Table 2.4 and Table 2.5,respectively.

\begin{tabular}{|c|c|c|}
\hline Percentage of widthwise delamination & S.Kumar $(\mathrm{Hz})$ & FEA results(Hz) \\
\hline 0 & 81.46 & 81.409 \\
30 & 81.32 & 80.930 \\
50 & 80.15 & 80.199 \\
70 & 78.01 & 79.098 \\
90 & 76.12 & 78.994 \\
100 & 76.04 & 75.434 \\
\hline
\end{tabular}

Table 2.4: C-type layer delamination 


\begin{tabular}{|c|c|c|}
\hline Percentage of widthwise delamination & S.Kumar $(\mathrm{Hz})$ & FEA results $(\mathrm{Hz})$ \\
\hline 0 & 81.46 & 81.409 \\
30 & 81.51 & 80.34 \\
50 & 81.12 & 81.19 \\
70 & 80.31 & 80.94 \\
90 & 80.48 & 80.69 \\
100 & 68.24 & 64.69 \\
\hline
\end{tabular}

Table 2.5: I-type layer delamination

\subsubsection{Multiple delamination}

The same FE procedure was extended to perform modal analysis for the case of multiple delamination. As shown in the Fig 2.6, two delamination were introduced at different location in the middle surface of the $[0]_{4}$ laminate. Each delamination is of the full widthwise and $24 \mathrm{~mm}$ in lengthwise direction.

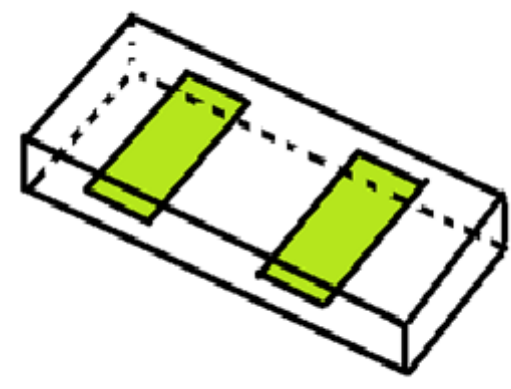

Figure 2.6: Multiple delamination

\begin{tabular}{|c|c|c|}
\hline Percentage of widthwise delamination & S.Kumar $(\mathrm{Hz})$ & FEA results $(\mathrm{Hz})$ \\
\hline First mode & 81.46 & 80.85 \\
Second mode & 509.124 & 484.6 \\
Third mode & 1421.05 & 1401.6 \\
Fourth mode & 2772.05 & 2546.2 \\
Fifth mode & 4555.83 & 3546.4 \\
\hline
\end{tabular}

Table 2.6: I-type layer delamination

\section{5 summary}

In this chapter, finite element modeling procedure for the simulation of partial delamination in composites was presented. FEA model of I-type, C-type, multiple delamination were modeled and studied using Ansys14. Modal analysis results of the composite beam show the change in natural frequency for the different types of delamination. The modal parameters i.e. frequency and mode shapes results are compared with the available literature results. These finite element modeling 
results of partial delamination needs to be validated by performing experiments which are discussed in the subsequent chapters. 


\section{Chapter 3}

\section{Vibration analysis of composite structure}

\subsection{Introduction}

Vibration based health monitoring techniques are widely used for real time monitoring of engineering structures. Doebling et al [9] discussed about the practical challenges regarding the implementation of vibration based SHM and the measurement systems available for the detection, location and quantification of damages from the acquired modal response. Vibration based SHM has gradually moved from accelerometers for sensing to embedded piezo sensors, fiber optic sensors and noncontact measurements. In this work, laser Doppler vibrometer (LDV) is used for studying the modal response of delaminated composite structures and the details of the experimental setup for composite material and damage characterization is presented.

\subsubsection{Theory and specification of LDV}

Laser Doppler Vibrometer (LDV) consists of a scanning head which is capable of measuring the movements of the scanning points in Z-direction i.e out of plane displacement. It is a non-contact vibration measurement technique. The system works on the principle of Doppler Effect and interferometry for vibration measurement. The minimum detectable vibration speed using this system is $100 \mathrm{~mm} / \mathrm{s}$ at $1 \mathrm{~Hz}$ resolution while the maximum speed is of $10 \mathrm{~m} / \mathrm{s}$. Application of LDV includes modal analysis of automotive parts, car bodies and air craft panels etc. Fig. 3.1 indicates the usage of LDV in automobile industries.

\subsection{Principle of Doppler Effect}

Doppler Effect is the change in the frequency or wavelength of emitted waves as the source of the wave approaches or moves away from an observer. It was named after the Austrian physicist Christian Johann Doppler. The change or shift in frequency observed depends on the speed and direction of travel of both source and observer. Helium-Neon (He-Ne) Laser beam $(\lambda=316 \mathrm{~nm})$ is made to incident on the vibrating surface and the reflected Laser light from the surface was detected by the 


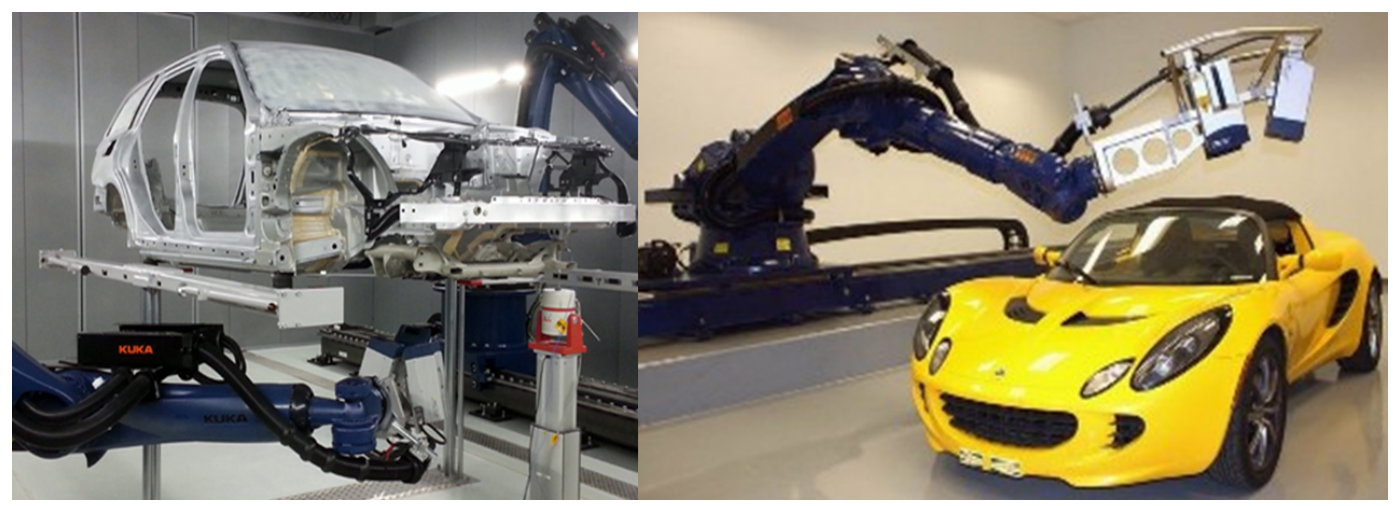

Figure 3.1: Laser Doppler vibrometer is used perform the modal analysis on the car bodies

vibrometer scanning unit. Incident and reflected beams are made to interfere on the detector by suitable arrangement. A moving surface induces a frequency shift on the light received by vibrometer optics.

$$
f_{D}=\frac{2 V}{\lambda}
$$

where $f_{D}$ is the frequency shift in the reflected beam, $V$ is the velocity of specimen surface and $\lambda$ is the wavelength of He-Ne laser. Laser Doppler Vibrometer works on the basis of optical interference requiring two coherent light beams. The interference term relates to the path difference between both the beams. If the path difference between the interfering beams is integral multiplier of Laser wavelength, constructive interference occurs else destructive interference occur.

$$
I_{\text {total }}=I_{1}+I_{2}+2 \sqrt{I_{1} I_{2}} \cos \left[\frac{2 \pi\left(r_{1}-r_{2}\right)}{\lambda}\right]
$$

where $I_{\text {total }}$ is the resultant intensity, $I_{1}$ and $I_{2}$ are the intensities of two interfering laser beams and $\left(r_{1}-r_{2}\right)$ is the path difference. In this case, overall intensity becomes four times the single intensity. If the path difference is odd multiplier of half the wave length, destructive interference occurs where the overall intensity becomes zero. The interference phenomenon in Laser Doppler Vibrometer is shown in the Fig. 3.2. Beam Splitter 1 direct the inference beam toward beam-splitter

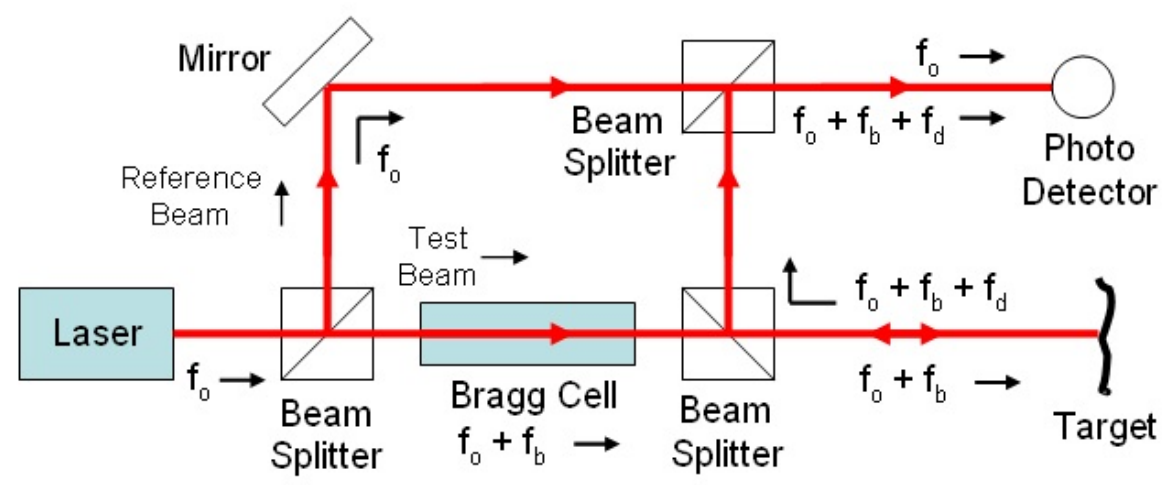

Figure 3.2: Basic Principle of L.D.V 
2. After passing the beam from beam-splitter 2, the measurement beam is focused on the object to be measured. The object to be investigated must be reflective. Surface of the object may be made reflective by applying Ard-oxspray coating or retro-reflective tape. The reflected beam is deflected by second beam-splitter and merges with the reference beam by the third beam-splitter and then it directed on to the photo-detector. As the path length of their inference beam is constant overtime, a movement of object under consideration generates a dark and bright fringe pattern on the detector. One complete dark bright cycle corresponds to an object displacement of exactly half the wavelength of the light used. For a He-Ne Laser, this displacement is 316 nanometers. Change in the optical path length per unit time causes the Doppler frequency shift of the measured beam. The modulation frequency of the interferometer pattern is exactly proportional to the velocity of the object. Interference patterns (and frequency shifts) are generated as the object moves towards or moves away from the interferometer. A Bragg cell is placed in the inference beam to distinguish the direction of movement as it shifts the Laser frequency by $40 \mathrm{MHz}$. A modulation frequency of the fringe pattern of $40 \mathrm{MHz}$ is generated when the object is at rest. Movement of the object towards the interferometer reduces the modulation frequency while it increases frequency lower or higher than $40 \mathrm{MHz}$ indicating the direction and amplitude of movement of the object.

\subsection{Experimental modal mnalysis using LDV}

Modal parameters associated with corresponding natural frequencies and mode shapes obtained from experimental technique using LDV describes the dynamic properties of structure. The experimental modal analysis involves measurement of time-domain data, which transform time-domain data in to frequency domain-data such as frequency response functions (FRFs) using Fouriers transform, and FRF analysis. The experimental modal analysis was conducted using a Polytec PSV-500 non-contact scanner of LDV and data was processed using software PSV9.0 to extract the natural frequencies and corresponding mode shapes. The schematic diagram for LDV setup is shown in Fig. 3.3. The input signal is given to scanning head from vibrometer controller of the LDV system and another external signal is given to power amplifier for excitation purpose of the specimen. For the dynamic excitation of the composite beam an electro-dynamic shaker (IMV, VE-7144) issued. A power amplifier (IMV, MODELCE-7144) is connected to the shaker for the purpose of amplifying the excitation signal generated by the LDV system.

\section{Experimental setup}

Composite beams are coated with white spray for improving the reflection properties. The beam was fixed on one end in a fixture which is mounted on a shaker as shown in the Fig 3.3. A periodic chirp signal voltage of $10 \mathrm{~V}$ with frequency range $0-5000 \mathrm{~Hz}$ was used to excite the shaker. The detector or receiver was able to measure instantaneous velocity in $\mathrm{mm} / \mathrm{s}$ and increased amplifier gain was used to extract the higher frequency modes.

\section{Alignment procedure}

The 2-D alignment step is carried out to relate the video and scanner coordinate system. This is done by assigning 10-15 points on the scanning area through laser scanning head. PSV software 


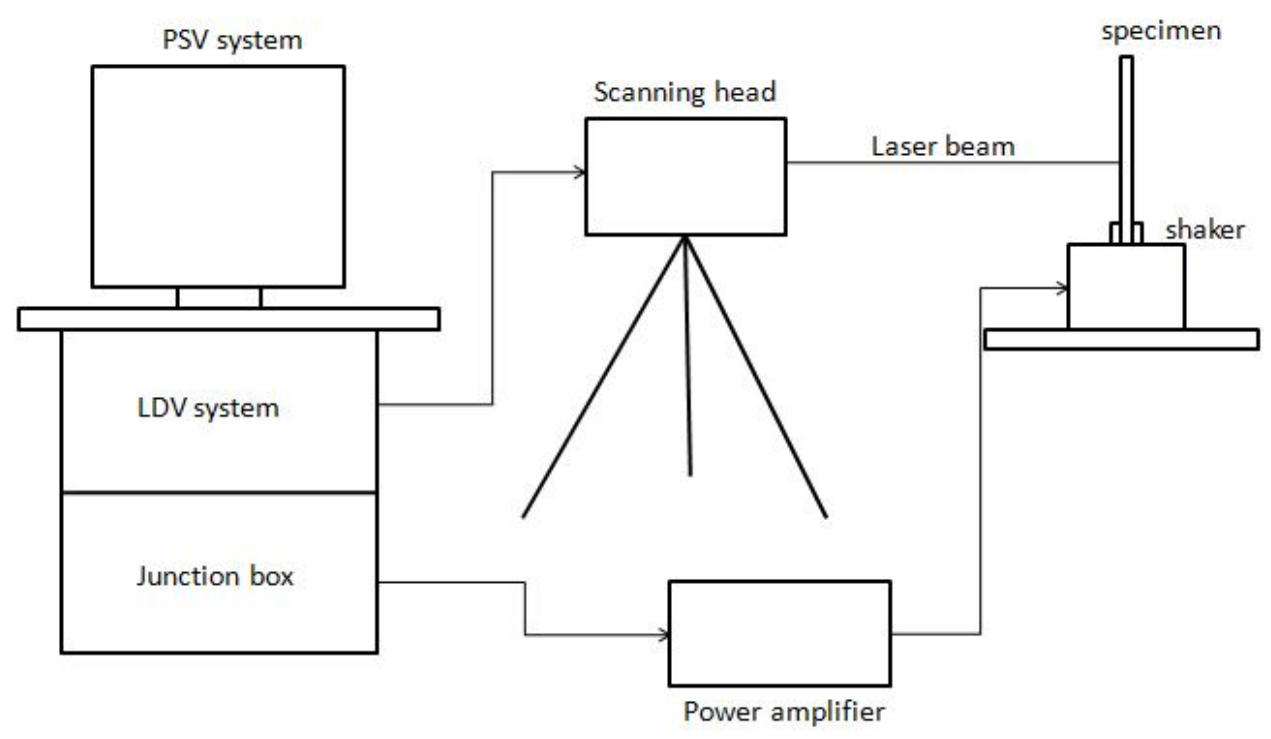

Figure 3.3: Setup of L.D.V

stores the video coordinates and the scan angles of the points on the scan area and calculates the polynomial interpolation. The 2-D alignment step performed for one of the scan heads is shown in Fig. 3.3. Surface of the specimen should be perpendicular with the laser scanning head.

\section{Geometry scan}

All the scan points in the mesh grid area accessed by the Laser in order to estimate the surface of the test object. The Laser coincides at each and every scan point on the mesh grid. The accuracy of the surface estimated depends on the previous steps. This step is crucial to obtain the exact simulations of the test surface during the test.

\section{Data Acquisition parameters}

Data acquisition properties such as the excitation signal to be used, frequency range, parameters for the FFT analysis (number of FFT lines are 6400, bandwidth $5 \mathrm{kHz}$ ), average magnitude is 3, velocity decoder are given in this step. Vibration velocity used is $500 \mathrm{~mm} / \mathrm{s}$. Time required for the complete scan depends on the number of scanning points defined on the specimen and FFT parameters. Response plots, mode shapes animations are visualized after the scan in present at on mode.

\subsubsection{Specimen Fabrication}

In this work, glass fiber reinforced plastic (GFRP) material is chosen for the experimental modal analysis. The GFRP specimens were fabricated using hand layup and vacuum bagging technique. Glass fiber Sika (SG930) fiber-mat material and Epoxy resin (CY-230) is taken in 1:1 proportion and hardener HY-951 (Huntsman) is taken as 1:10 proportion as that of epoxy resin. In this technique, 
resin is applied between each layer of fiber mat and above which perforated sheet and peel ply layer is placed for good surface finish. Subsequently, breather material is placed on top for removing excess resin by suction. The setup is enclosed by pasting seal end-tape over which vacuum bag was applied. At the center of the vacuum bag, a flange was inserted which is further connected to vacuum pump.

\subsection{Material characterization of composite structure}

Grediac and Paris. [38] proposed a dynamic method for the characterization of the composite structure. For removing the noisy data. Grediac and Vautrin [39] suggested the use of special optical devices during modal testing of structures. Later Batista et al [41] introduced the usage of regressive discrete Fourier series (RDFS) for removing the noisy data. RDFS was applied to smooth experimental mode shapes and consequently to reduce noise. Elastic constants are then computed by following the procedure proposed by Grediac and Paris. [38]. Second-order spatial partial derivatives of mode shapes are computed by finite differences from the smoothed shapes. Numerical and experimental tests were carried out to assess the accuracy of the proposed method by Batista et al - [41]. It was shown that the method compute the elastic constant accurately, even when the input data contains noise. It was direct method to determine the six flexural stiffnesses of thin anisotropic plates. This technique was based on a relevant processing of natural frequencies and mode shapes of free-free vibrating plates. The unknown plate rigidities are the solution of an overdetermined linear system. The equations which are obtained with a set of relevant weighting functions associated with different natural modes of vibration. In this work, the approach given by Grediac and Paris was used to determine the elastic properties of GFRP specimens.

\subsubsection{Governing differential equation of plate}

The partial differential equation governing the transverse vibration of a free-free symmetrically laminated thin plate is given by,

$$
D_{11} \frac{\partial^{4} w}{\partial x^{4}}+4 D_{16} \frac{\partial^{4} w}{\partial x^{3} \partial y}+2\left(D_{12}+D_{66}\right) \frac{\partial^{4} w}{\partial x^{2} \partial y^{2}}+4 D_{16} \frac{\partial^{4} w}{\partial x \partial y^{3}}+D_{22} \frac{\partial^{4} w}{\partial y^{4}}=-\rho h \frac{\partial^{2} \omega}{\partial t^{2}}
$$

where $w$ is the transverse displacement and $D_{i j}$ is the bending stiffness matrix of the laminate. To characterize the composite material, the $D_{i j}$ matrix have to be determined from the modal analysis. To solve for the six different unknowns, first six mode shapes are required from the modal analysis. But the drawback with this type of method is the sensitivity of the curvature of higher mode value to experimental noise and this leads to error in stiffness constant evaluation. Therefore to avoid this problem, the governing equation was multiplied with weighting function $v(x, y)$ such that some of the coefficients in the governing equation vanishes.

\subsubsection{Rules for the selection of the weighting function}

Equation 3.3 is valid for any mode shape and weighting function $v$. One can therefore build up a set of linear equations in which the stiffness coefficients are unknown. However few rules must be applied for choosing weighting function. For instance, weighting function depends either on $x$ or $y$ coordinate and results in three of the six coefficients in equation 3.3 to be zero. Hence one can 
obtain partially uncoupled equations by appropriately choosing the mode and weighting function. For example, modal analysis was carried out on a unidirectional composite plate of $200 \mathrm{~mm} \times 200 \mathrm{~mm}$ and thickness of $1 \mathrm{~mm}$ and the mode shapes obtained are shown in Fig. 3.4. The integral or weak

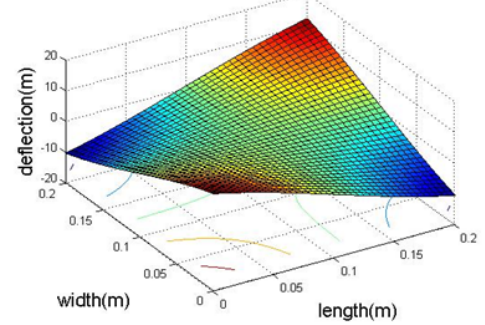

a)

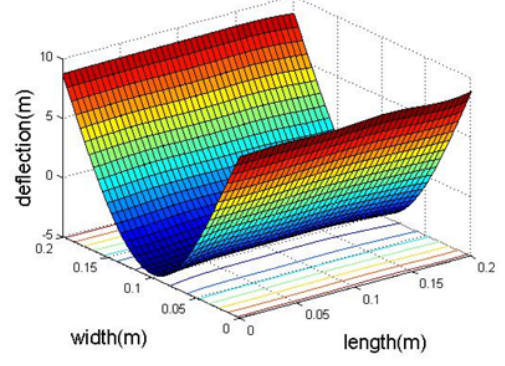

b)

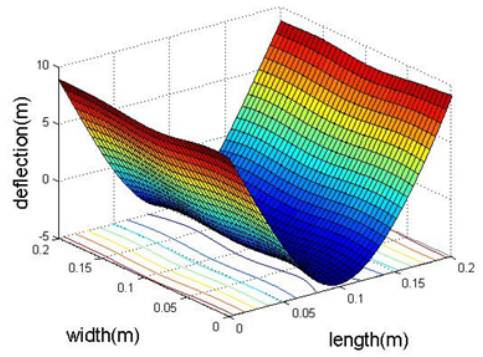

c)

Figure 3.4: a) 1st mode shape b) 2nd mode shape c) 5 th mode shape

form of the plate is given by,

$$
\begin{array}{r}
D_{11} \int_{s} \frac{\partial^{2} w}{\partial x^{2}} \frac{\partial^{2} v}{\partial x^{2}} d s+D_{22} \int_{s} \frac{\partial^{2} w}{\partial y^{2}} \frac{\partial^{2} v}{\partial y^{2}} d s+D_{12} \int_{s}\left(\frac{\partial^{2} w}{\partial y^{2}} \frac{\partial^{2} v}{\partial x^{2}}+\frac{\partial^{2} w}{\partial x^{2}} \frac{\partial^{2} v}{\partial y^{2}}\right) d s+4 D_{66} \int_{s}\left(\frac{\partial^{2} w}{\partial x \partial y} \frac{\partial^{2} v}{\partial x \partial y}\right) d s \\
+2 D_{16} \int_{s}\left(\frac{\partial^{2} w}{\partial x \partial y} \frac{\partial^{2} v}{\partial x^{2}}+\frac{\partial^{2} w}{\partial x^{2}} \frac{\partial^{2} v}{\partial x \partial y}\right) d s+2 D_{26} \int_{s}\left(\frac{\partial^{2} w}{\partial x \partial y} \frac{\partial^{2} v}{\partial y^{2}}+\frac{\partial^{2} w}{\partial y^{2}} \frac{\partial^{2} v}{\partial x \partial y}\right) d s=-\rho h \int_{s} \frac{\partial^{2} \omega}{\partial t^{2}} v d s
\end{array}
$$

The assumed solution for this governing equation is given by,

$$
\omega_{i}=W^{i} \sin \left(\omega_{i} t\right)
$$

where $W$ is the deflection amplitude of mode shape and $\omega$ is the corresponding natural frequency. Three independent quadratic functions were used to decouple the equation,

1) $v_{1}(x, y)=x^{2}$

$$
D_{11} K_{x x}^{i}+D_{12} K_{y y}^{i}+2 D_{16} K_{x y}^{i}=\frac{\rho h}{2} \omega_{i}^{2} \int_{s} W_{i} x^{2} d s
$$

2) $v_{1}(x, y)=y^{2}$

$$
D_{11} K_{y y}^{i}+D_{12} K_{x x}^{i}+2 D_{26} K_{x y}^{i}=\frac{\rho h}{2} \omega_{i}^{2} \int_{s} W_{i} y^{2} d s
$$


3) $v_{1}(x, y)=x y$

$$
2 D_{66} K_{x y}^{i}+D_{16} K_{x x}^{i}+D_{26} K_{y y}^{i}=\frac{\rho h}{2} \omega_{i}^{2} \int_{s} W_{i} x y d s
$$

where

$$
K_{x x}^{i}=\int_{s} \frac{\partial^{2} w}{\partial x^{2}} d s K_{y y}^{i}=\int_{s} \frac{\partial^{2} w}{\partial y^{2}} d s K_{x y}^{i}=\int_{s} \frac{\partial^{2} w}{\partial x y} d s
$$

After decoupling the equation, a matrix form of above three equation is given by.

$$
\left[\begin{array}{llllll}
k_{x x}^{i} & 0 & k_{y y}^{i} & 0 & 2 k_{x y}^{i} & 0 \\
0 & k_{y y}^{i} & k_{x x}^{i} & 0 & 0 & 2 k_{x y}^{i} \\
0 & 0 & 0 & 2 k_{x y}^{i} & k_{y y}^{i} & k_{y y}^{i}
\end{array}\right]\left[\begin{array}{c}
D_{11} \\
D_{22} \\
D_{12} \\
D_{66} \\
D_{16} \\
D_{26}
\end{array}\right]=\frac{-\rho h \omega_{i}^{2}}{2}\left[\begin{array}{l}
\int W_{i} x^{2} d s \\
\int \\
\int_{s} W_{i} y^{2} d s \\
\int \\
s
\end{array}\right.
$$

\subsubsection{Selection of mode shape}

The first mode of vibration is mainly twisting and it strongly depends on $D_{66}, D_{16}$ and $D_{26}$. In the same way the second mode of vibration depends on bending along direction 1. i.e. it strongly depends on $D_{22}, D_{12}$ and $D_{26}$. Finally the fifth mode of vibration is mainly bending along direction 2 and it strongly depends on $D_{11}, D_{12}$ and $D_{16}$. In conclusion, the six unknowns are directly and strongly involved in these three basic modes. In contrast the set of modes 2,4 and 9 as shown in fig. 3.5 would not allow for determination of the six unknowns as these three modes are mainly bending along direction 1 only. The final form of the matrix equation given by,

$$
A \cdot D=B
$$

where $A$ is a $3 p \times 6$ matrix, $D$ is a $6 \times 1$ matrix and $B$ is a $3 p \times 1$ matrix. The least squares method was used to solve the matrix equation and the stiffness coefficients are evaluated using the expression,

$$
D=\left(A^{t} A\right)^{-1}\left(A^{t} B\right)
$$

The above approach when extended to experimental results require the mode shapes to be smooth and free from noise. The reason being evaluation of curvatures of mode shapes is highly sensitive to the noise and might leads to erroneous results. Therefore, pre-processing operations of the mode shapes have to be performed to remove the unwanted noise and is explained in the next section.

\subsection{The Regressive fourier series}

In this work, a smoothing technique proposed by Arruda [46] called regressive discrete Fourier series (RDFS) was applied to remove the noise and smooth the mode shapes. RDFS uses least squares to estimate the coefficients of a two-dimensional discrete Fourier series with arbitrary periods and 


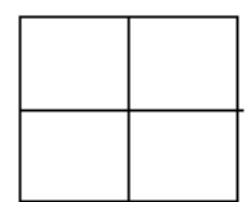

1st mode

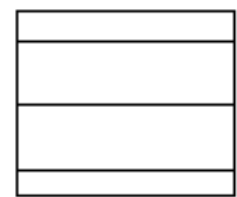

4th mode

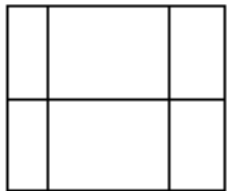

7th mode

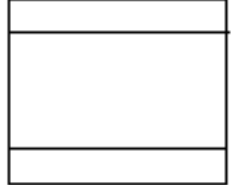

2nd mode

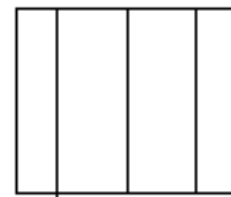

5 th mode

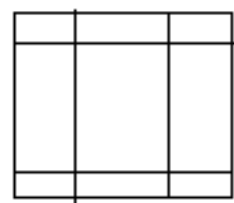

8th mode

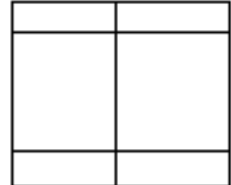

3rd mode

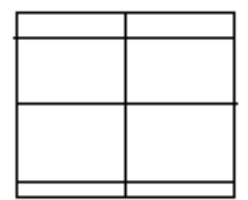

6 th mode

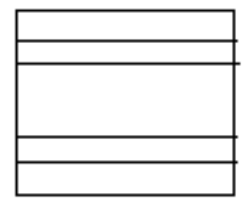

9th mode

Figure 3.5: Different mode shapes

arbitrary frequency resolutions [46]. The RDFS is expressed as:

$$
x_{m n}=\sum_{k=-p}^{p} \sum_{l=-q}^{q} X_{k l} W_{R}^{m k} W_{C}^{l n}+\epsilon_{m n} m=0, \ldots \ldots, M-1 ; n=0, \ldots \ldots . N-1
$$

where $M$ and $N$ are the data size i.e number of row and column, $R$ and $C$ are the period size of the discrete Fourier series, rows and column respectively, $W_{R}=\exp (-i 2 \pi / R)$ and $W_{C}=\exp (-i 2 \pi / C)$ are the $R^{t h}$ and $C^{t h}$ root of unity, $X_{k l}$ are the two-dimensional Euler-Fourier coefficients, $\epsilon_{m n}$ is the element of error matrix and $P, Q$ are the number of frequency lines. The number of frequency line kept less than the data size. Thus an approximation is made and the Euler-Fourier coefficients can be computed using least square method. The equation 3.12 written in matrix form given by

$$
x=W_{R} X W_{C}+\epsilon
$$

where $\epsilon$ is error vector in arbitrary domain RDFS. The least square solution is given by

$$
X=\left(W_{R}^{H} W_{R}\right)^{-1} W_{R}^{H} x W_{C}^{H}\left(W_{R}^{H} W_{R}\right)^{-1}
$$

The superscript $h$ denotes the complex conjugate transpose of a matrix. It should be noted that matrices to be inverted have a very small size,i.e. $(2 p+1 \times 2 p+1)$ and $(2 q+1 \times 2 q+1)$, respectively. The smoothed shape of the mode shape was obtained by putting the value of Euler-Fourier Coefficients back into equation number 3.15 .

$$
x^{s}=W_{R} X W_{C}
$$


The various steps involved in evaluating the elastic properties of the material using modal parameters is given in the flowchart fig. 3.6. For the validation of the algorithm, a isotropic steel plate with

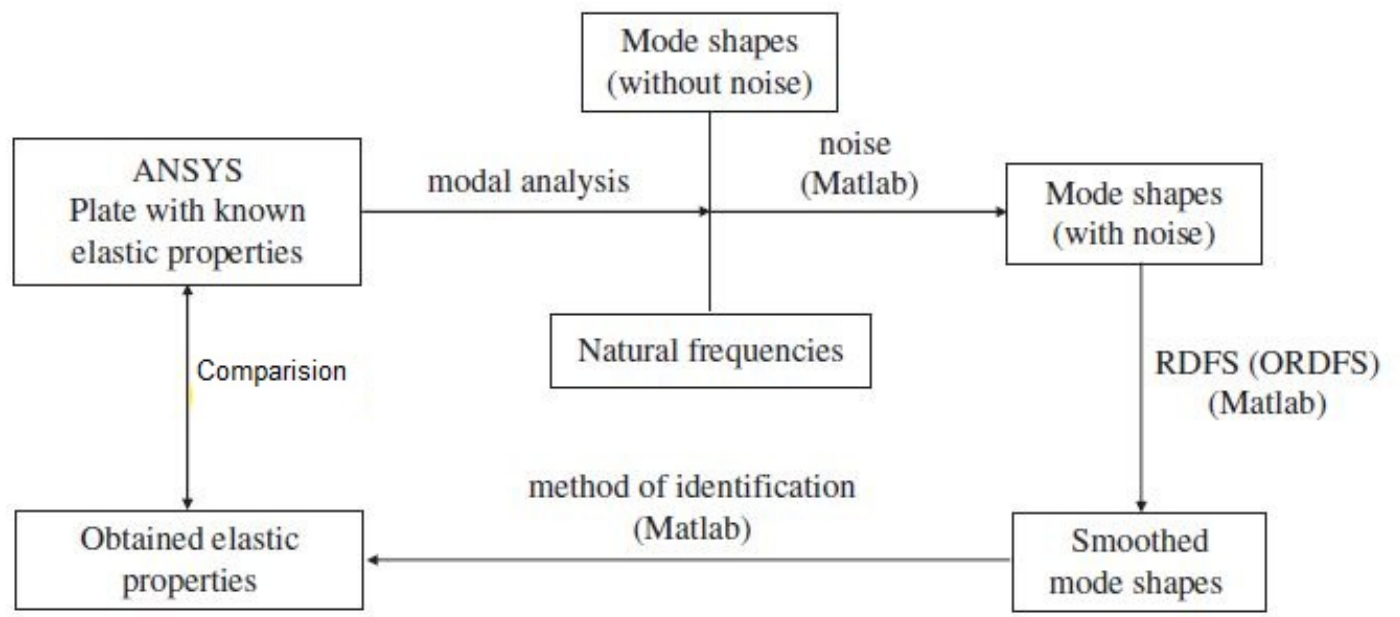

Figure 3.6: Numerical identification process of the elastic properties flow chart

$E_{11}=210 \mathrm{GPa}, \gamma=0.3, G_{12}=80.8 \mathrm{GPa}$ was chosen for the numerical study. The dimension of the steel plate is taken as $200 \mathrm{~mm} \times 200 \mathrm{~mm} \times 1 \mathrm{~mm}$. Shell element SHELL181 was used to model the plate and modal analysis under free-free boundary condition was performed. For sake of checking the regressive discrete Fourier series we introduce noise into the mode shape data as shown in fig. 3.7. These mode shapes were then evaluated using RDFS and smoothed mode shapes are given in Fig. 3.8. Obtained frequency for the steel plate are $84.127 \mathrm{~Hz}$ for the 1st mode i.e. twisting mode, 122.51 Hz for the 2nd mode i.e. bending along $\mathrm{y}$-axis, $151.79 \mathrm{~Hz}$ for the $3 \mathrm{rd}$ mode i.e. bending along x-axis. And after performing the R.D.F.S. we get the smooth mode shape as follow After extracting the smoothed mode shape, the algorithm of method of identification of material properties was used to determine the bending stiffness of the isotropic plate and the results are shown in following table 3.1. Next, the algorithm was applied to determine the bending stiffness coefficients of the

\begin{tabular}{|c|c|c|c|}
\hline Bending Stiffness & Analytical values $(\mathrm{GPa})$ & FEA values(GPa) & error percentage \\
\hline$D_{11}$ & 19.2308 & 19.2835 & 0.273 \\
$D_{22}$ & 19.2308 & 19.2835 & 2.1285 \\
$D_{12}$ & 5.7692 & 5.6466 & 0.273 \\
$D_{66}$ & 6.7333 & 6.7397 & 0.095 \\
$D_{16}$ & 0 & 0 & 0 \\
$D_{26}$ & 0 & 0 & 0 \\
\hline
\end{tabular}

Table 3.1: Bending stiffness of Steel plate(isotropic material)

composite plate [38]. Elastic properties are given by $E_{11}=120 \mathrm{GPa}, E_{22}=10 \mathrm{GPa}, G_{12}=4.9 \mathrm{GPa}$, $12=0.3$, Density $=1510.0 \mathrm{~kg} / \mathrm{m} 3$. After performing modal analysis on the composite plate, we get the frequency as $47.633 \mathrm{~Hz}$ for the 1st mode, 66.204 for the 2nd mode, and for the 3rd mode was $229.8 \mathrm{~Hz}$. And following table 3.2 shows the bending stiffness of composite plate. 

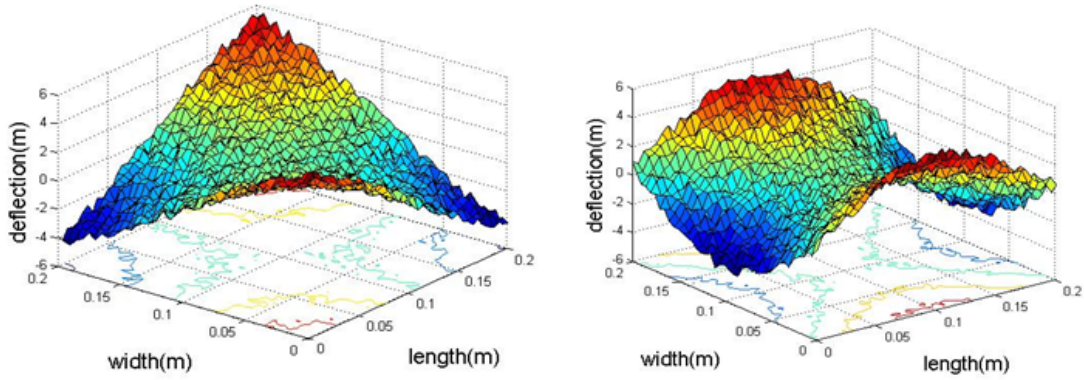

a)

b)

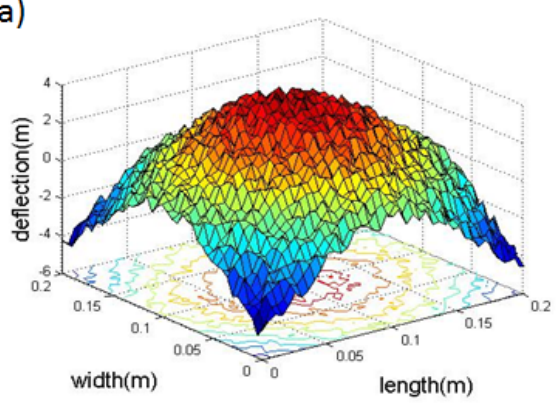

c)

Figure 3.7: a) 1st Mode shape with noisy data b) 2nd Mode shape with noisy data c) 3rd Mode shape with noisy data

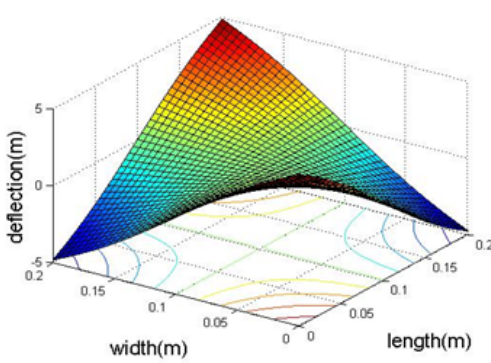

a)

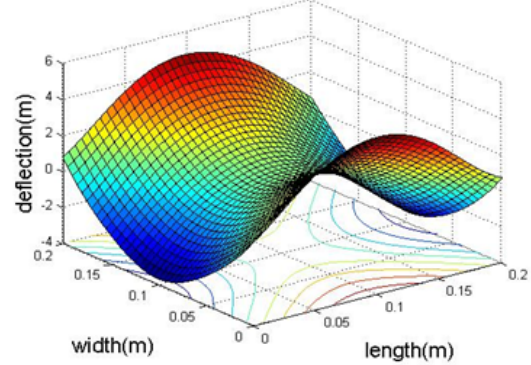

b)

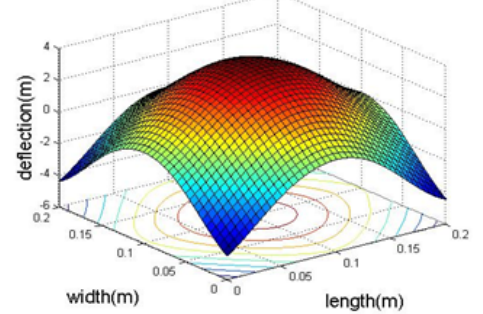

c)

Figure 3.8: a) $1^{\text {st }}$ modeshape of steel plate b) $2^{\text {nd }}$ modeshape of steel plate c) $3^{r d}$ modeshape of steel plate

\subsubsection{Experimental results for bending stiffness of the composite plate}

Experimental modal analysis on the composite plate $[0]_{4}$ having dimension as $200 \mathrm{~mm} \times 200 \mathrm{~mm} \times$ $1.16 \mathrm{~mm}$ was performed using LDV. Composite plate was hanged on the frame with help of the 


\begin{tabular}{|c|c|c|c|}
\hline Bending Stiffness & Analytical values $(\mathrm{GPa})$ & F.E.A values(GPa) & error percentage \\
\hline$D_{11}$ & 10.0756 & 10.1295 & 0.5 \\
$D_{22}$ & 0.8396 & 0.8442 & 0.5478 \\
$D_{12}$ & 0.2519 & 0.2460 & 2.3421 \\
$D_{66}$ & 0.4083 & 0.4088 & 0.12245 \\
$D_{16}$ & 0 & 0 & 0 \\
$D_{26}$ & 0 & 0 & 0 \\
\hline
\end{tabular}

Table 3.2: Bending stiffness of composite plate

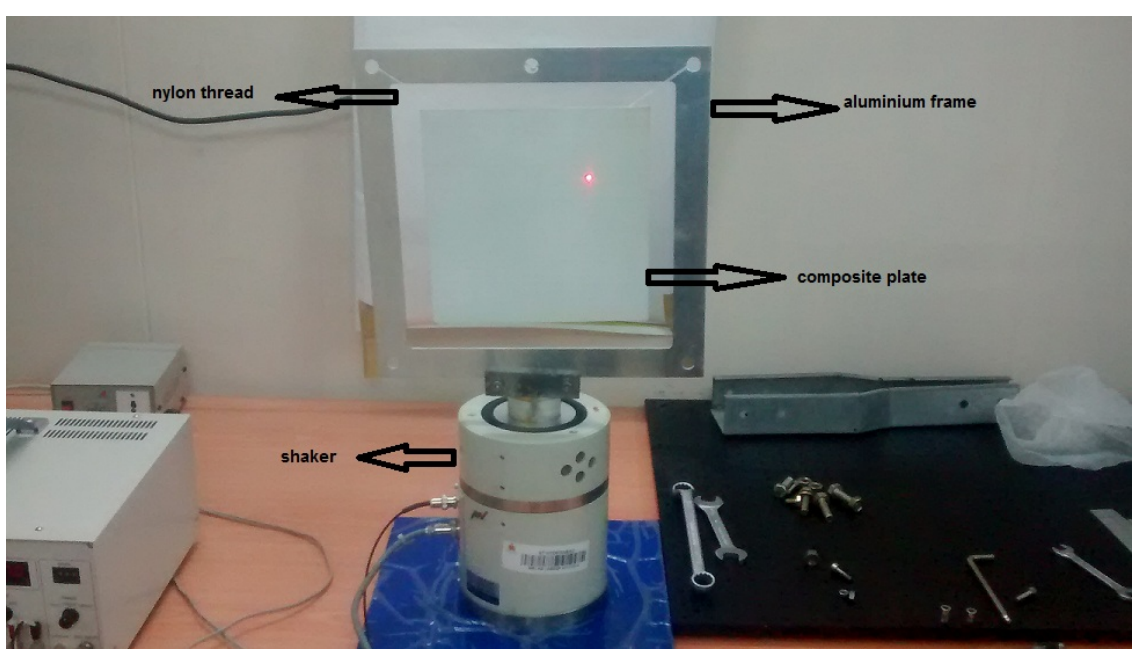

Figure 3.9: Experimental setup for characterization

nylon threads as shown in the fig. 3.9 to create the free-free boundary condition. Nylon threads were used to transfer vibration to the specimen. Following the same procedure in numerical studies, the bending stiffness properties obtained from experiments are given in table 3.3:

\begin{tabular}{|c|c|c|c|}
\hline Serial no. & Bending stiffness & By D.I.C test GPa & By vibration analysis GPa \\
\hline 1 & $D_{11}$ & 4.1485 & 4.1498 \\
2 & $D_{22}$ & 1.2217 & 1.1657 \\
3 & $D_{12}$ & 0.2443 & 0.2257 \\
4 & $D_{66}$ & 0.5836 & 0.5969 \\
5 & $D_{16}$ & 0 & 0.0007 \\
6 & $D_{26}$ & 0 & 0.0013 \\
\hline
\end{tabular}

Table 3.3: Bending stiffness of composite plate

\subsection{Summary}

In this chapter details of the experimental setup using LDV to carry out modal analysis of composite plates were presented. The procedures for material characterization using modal analysis is presented. RDFS algorithm was applied to smooth the experimentally obtained mode shapes and further used to evaluate the curvature of mode shapes. The smoothed mode shapes were used in the algorithm for evaluation of material properties of composite specimen. The elastic properties obtained from modal analysis matched well with the results from static destructive tests. 


\section{Chapter 4}

\section{Damage characterization approaches}

\subsection{Introduction}

A reliable and effective healthy monitoring technique should be able to locate and characterize the size of the damage present in the structure. The fundamental idea for vibration based damage identification method is that the damage induces changes in physical properties such as mass, damping, and stiffness which cause detectable change in modal parameters like natural frequency, modal shapes and mode curvature. Most of the damage detection methods require a baseline or healthy structure information for locating the damage in structure. In this work, fractal dimension technique which is a baseline free method was applied to locate and quantify the size of the delamination in composite structures.

\subsection{Literature review}

Damage identification methods can be categorized as either local or global damage identification techniques [9]. Local damage identification techniques, such as ultrasonic methods and X-ray methods, require that the vicinity of damage is known a priori and readily accessible for testing, which cannot be guaranteed for most cases in civil or aerospace engineering. Hence, the vibration-based damage identification method as a global damage identification technique is developed to overcome these difficulties. Doebling et al. [9] presented an extensive review of vibration-based damage detection methods up to 1996. Sohn et al. [47] then presented an updated version of this review on the literature up to 2001. In both the articles, the features extracted for identification were considered to classify the damage identification methods. Damage identification methods are categorized on the bases of natural frequency, mode shape methods, curvature/strain mode shape-based method, and other method based on modal parameters. Gudmundson [48] used an energy-based perturbation approach and derived an explicit expression for the resonance frequencies of a wide range of damaged structure. Liang et al. [49] addressed the issue of determining frequency sensitivity for simply supported or cantilevered beam with one crack and developed analytical relationships between the first-order changes in the eigen frequencies and the location and severity of the damage. Here 
critical limitation is that the frequency changes caused by damage are usually small and may be buried in the changes caused by environmental and operational condition. Pandey et al. [50] suggested for the first time that the mode shape curvature i.e. the second derivatives of mode shape, are highly sensitive to damage and can be used to localize it. The curvature mode shapes are derived using a central difference approximation. But this method works perfectly when the specimen having full width delamination, but it does not perform well to through width delamination. The traditional mode shape change method uses the mode shape change from intact and damaged structures as a basic feature for damage identification. The baseline data from intact structure can be obtained from either an experimental test on the intact structure or an accurate numerical model of the intact structure. The traditional mode shape change methods are not very sensitive to damage, and they are commonly subjected to several limitations. For example, they are only sensitive to damage in certain area of the structures (e.g., mid-span of a clamped-clamped beam), and without further signal processing or pattern recognition technique, they can only be adopted for preliminary damage localization rather than accurate localization or quantification of damage. Hadjileontiadis and Douka [35] proposed a response-based damage detection algorithm for beams and plates using fractal dimension (FD) approach. But for higher mode shapes, this method give misleading information. To overcome this shortcoming, Wang and Qiao [34] proposed a modified FD method termed generalized fractal dimension (GFD) method by introducing a scale factor ' $S^{\prime}$ in the FD algorithm.

\subsection{Frequency based method}

This method is generally preferable where there is complete damaged zone. Specimen should not contain the error due to the noise and external vibration. Basic principal used in this type of method is that, it depends on the change in natural frequency of the specimen. If there is decrease in the natural frequency of a scanning nodes then the damage is present. This method is quite sensitive to the void and cracks. In experimental perspective it recommended to use at least 3 scanning head, so that in post processing it takes the average value of operational deformation shape. More ever this method is also sensitive to the boundary condition. If cantilever beam used in experiments then there is chance of error at free boundary condition. Therefore this type of algorithm misleads to the presence of the crack at the free- boundary condition. But it works extremely well for the fixed-fixed boundary condition.

\subsection{Mode shape-based method}

The main reason for the popularity of natural frequencies as damage indicator is that natural frequencies are rather easy to determine with a high degree of accuracy. The existing methods give a proper estimation of moderate cracks. However, when the size of the crack is small then this method is fail to show any change in natural frequency. To overcome this difficulties related to natural frequencies, many researchers have been focused on utilizing changes in mode shapes. 


\subsubsection{Traditional mode shape change method}

The tradition mode shape change method uses the mode shape change from intact and damaged structure as a fabric feature for damage identification. The baseline data from intact structure can be obtained from either an experimental test on the intact surface or an accurate numerical model of the intact structure. When experience data on the intact structure is not available a finite element model is usually adopted to generate the base line data. In general, the traditional mode shape change methods are very sensitive to several limitation. For example, they are only sensitive to damage in certain area of the structure e.g mid-span of a clamped-clamped beam. An analysis were perform on the cantilever having delamination at the middle portion in Ansys14 as shown in the fig. 4.1. After overlapping the delaminated beam and healthy beam mode shapes, we are able to

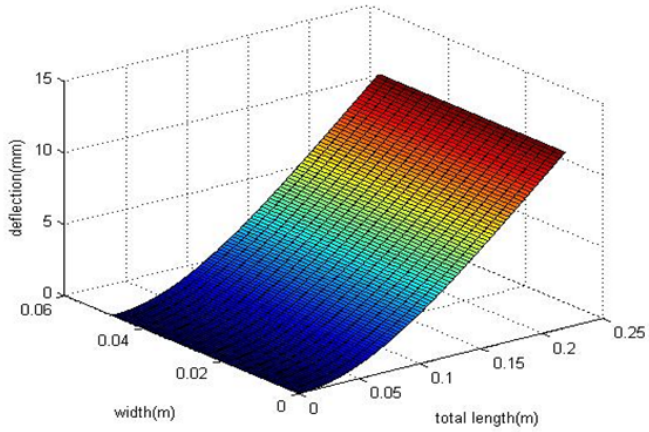

a)

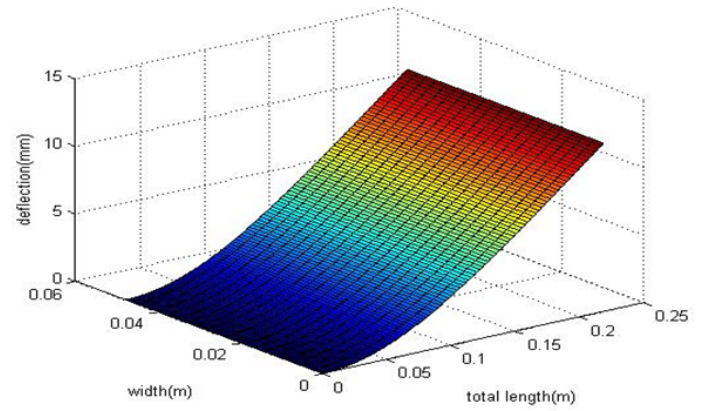

b)

Figure 4.1: a) $1^{\text {st }}$ mode of healthy specimen b) $1^{\text {st }}$ mode of delaminated specimen

distinguish the start of the delaminated region but not the end of the delaminated region. In the fig. 4.2. where red color region indicates the delaminated region. From above figure it is concluded

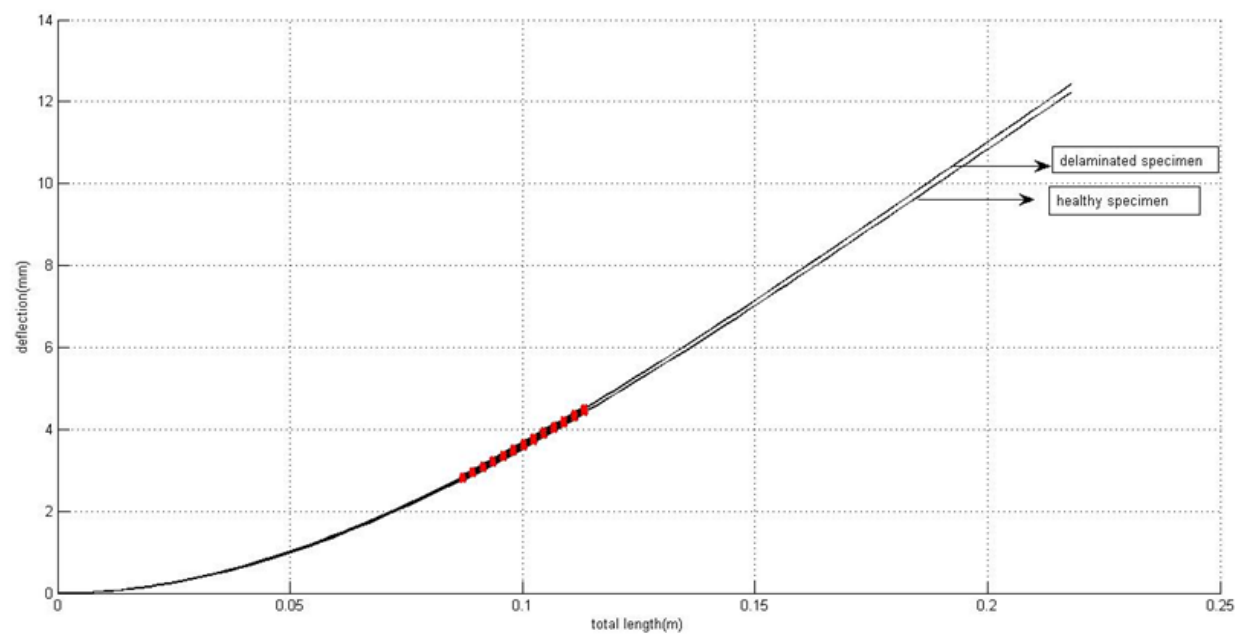

Figure 4.2: Overlap region of delaminated specimen and healthy specimen 
that the specimen having lower stiffness value will going to deflect more as compare to the healthy specimen.

\subsubsection{Curvature based method}

A particular vibration mode stores a certain amount of strain energy which is relatively sensitive to changes in the stiffness of the structure. The strain energy $\left(U_{i}\right)$ associated with a particular mode shape at 1 segment of the beam is calculated as

$$
U_{i}=\frac{1}{2} \int_{x_{k}}^{x_{k+1}} E I\left(\frac{\partial^{2} \phi}{\partial x^{2}}\right)^{2}
$$

where $\phi$ is the displacement of mode shape. EI is bending stiffness of the structure. Since bending stiffness and curvature in the strain energy, are interrelated, the damage induced specimen have reduction in stiffness which leads to change in the curvature. Due to the presence of noisy data in the operational deflection shape of the specimen, delamination is not able to distinguish properly. Therefore we use two techniques to minimize the error in data which were applied before finding the curvature of the specimen.

- RDFS technique

- Gapped smoothing technique

\section{RDFS}

RDFS is generally use in smoothing the signal and make it error free. But when we perform on the particular mode shape, then it clear error in the mode shape data and also the basic data of the delamination. Firstly we perform numerical simulation on the delaminated specimen, specimen having dimension as $220 \mathrm{~mm} 50 \mathrm{~mm}$ with delamination at the center of $24 \mathrm{~mm}$. After getting the mode shape, to reveal the data of delamination we need to find the curvature of the specimen. To check the reliability RDFS we introduce some noise in the mode shape and then apply RDFS. It clears the error which we introduce in the specimen but it also clears the delamination data. Therefore whatever the mode shape we are able to filter did not contains any data of the delamination. Numerical mode shape $\Rightarrow$ curvature of the mode shape Numerical mode shape $\Rightarrow$ introduce noise $\Rightarrow$ filter by RDFS $\Rightarrow$ curvature

\section{Gapped smoothing method}

The second derivative of mode shape are more sensitive to damage and can be used to localize it. The curvature mode shapes are derived using a central difference approximation as follow.

$$
\phi_{i}^{\prime \prime}=\frac{\left(w_{i+1}+w_{i-1}-2 w_{i}\right)}{h^{2}}
$$

where $w$ is the modal displacement at $i$-th point,$h$ is the sensor spacing. Results shows that the difference of curvature mode shape from intact and damaged structure can be good indicator of damage location. But due to the presence of noisy data can influence their results. Therefore to remove the noisy data, we need to filter it by gapped smoothing method. Gapped smoothing 


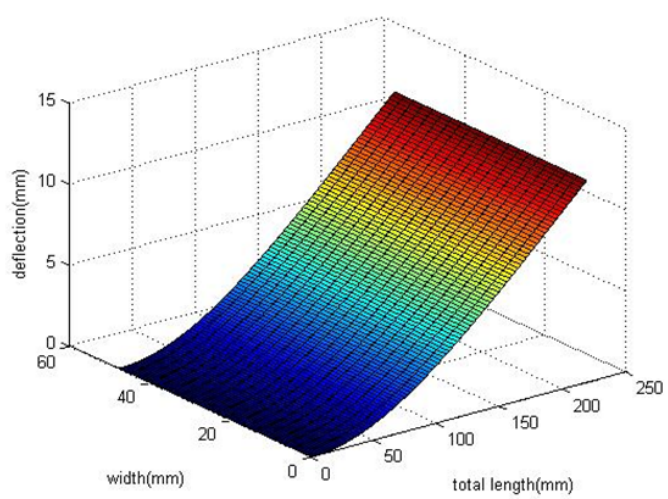

a)

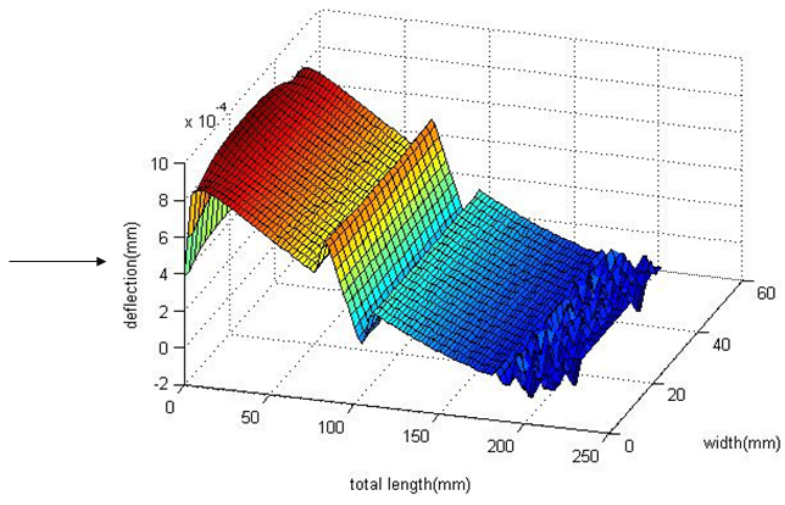

b)

Figure 4.3: a) $1^{\text {st }}$ mode of delaminated beam b) Curvature of $1^{\text {st }}$ mode

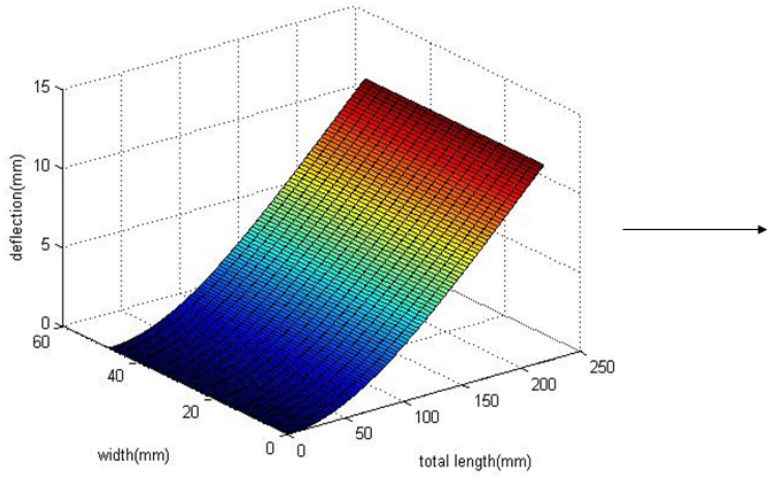

a)

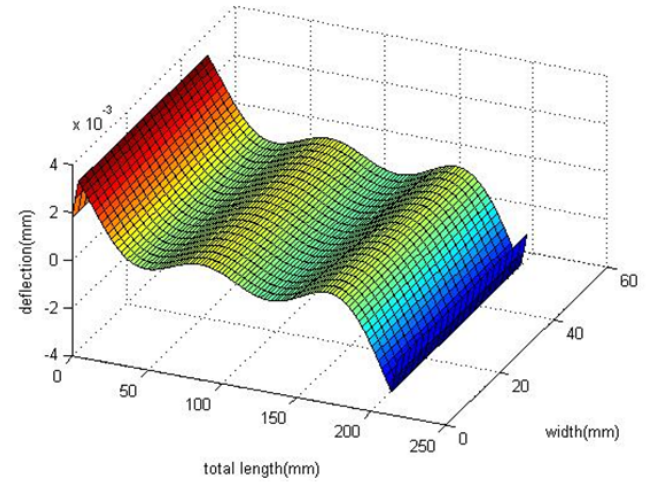

b)

Figure 4.4: a) $1^{\text {st }}$ mode of delaminated beam b) Curvature of $1^{\text {st }}$ mode after performing RDFS

method clear the noisy data up to certain limits such that it did not affect the basic information of the damage location. Gapped smoothing technique (GSM) is fitting of the cubic polynomial curve to the modal curvature change as mentioned in the equation '4.3. Given the curvature data $\phi$.

$$
\phi^{\prime \prime}=a_{0}+a_{1} x+a_{2} x^{2}+a_{3} x^{3}
$$

To the curvature data, using the four values of curvature are $\left(\phi_{i-2}^{\prime \prime}, \phi_{i-1}^{\prime \prime}, \phi_{i+1}^{\prime \prime}, \phi_{i+2}^{\prime \prime}\right)$ on the either side of the point $x_{i}$ to determine the value of the four unknown coefficient $a_{0}, . ., a_{3}$. The value are then used to calculate the value of $\phi_{i}=\phi\left(x_{i}\right)$. This procedure is repeated for all points $x_{i}, \mathrm{i}=$ $3, \ldots, \mathrm{N}-3$ as shown in the systematic diagram of curvature of the beam in fig. 4.5. From the fig.4.6, it is clearly visible to detect the presence of the delamination in the specimen. The effect of the noise gets decreases but not completely vanishes. Gapped smoothing technique where perform on the experimental output on various delaminated beam. But due to the error present at the free end of cantilever beam, only full width delaminated specimen and C-type delaminated specimen is able to detect the delamination as shown in the fig. 4.7. Red color indicates the delaminated portion. 


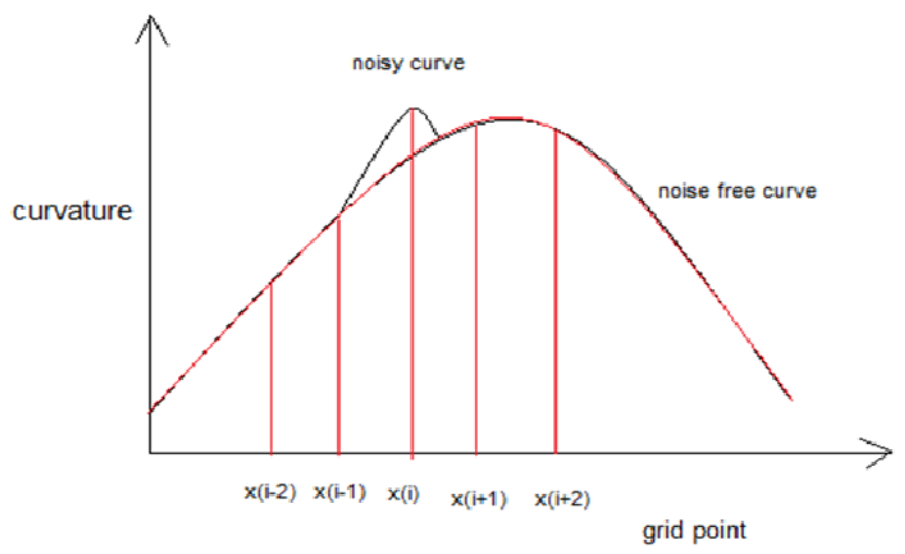

Figure 4.5: Gapped smoothing Technique

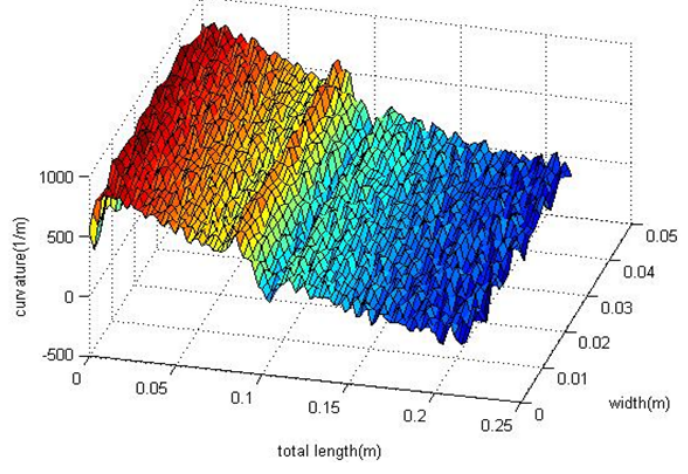

a)

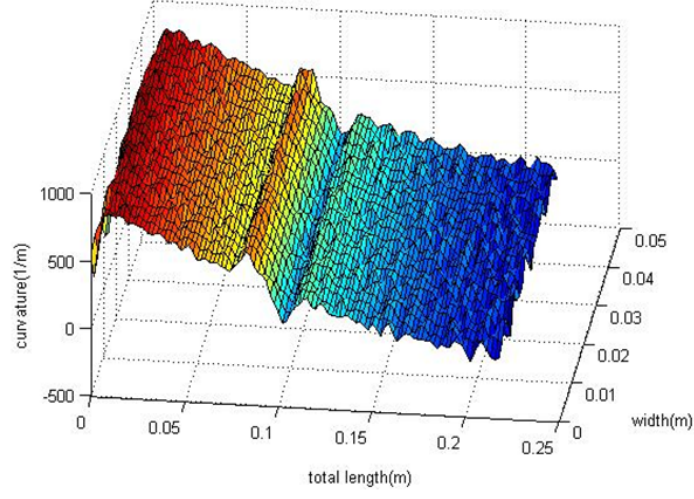

b)

Figure 4.6: a) $1^{\text {st }}$ mode curvature with noisy data b) $1^{\text {st }}$ mode curvature after performing GSM

Recently, signal processing techniques like continuous wavelet transform CWT is used for proper detection of the damage in the composite structure [42]. But the drawback related with the CWT is that, it require high density of sensors due to their natural difficulty to process a low number of sampling points.[42]. 


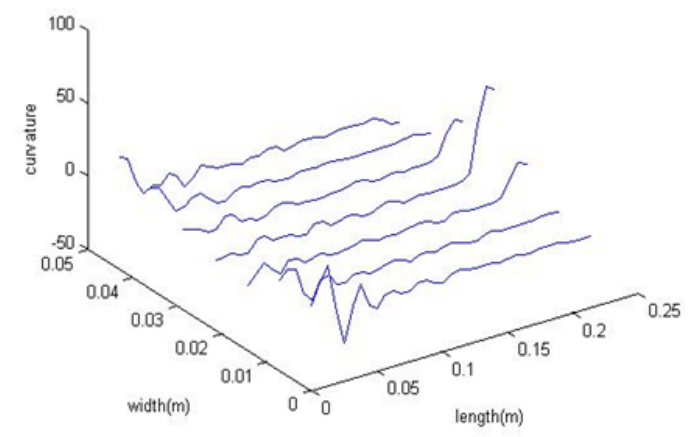

a)

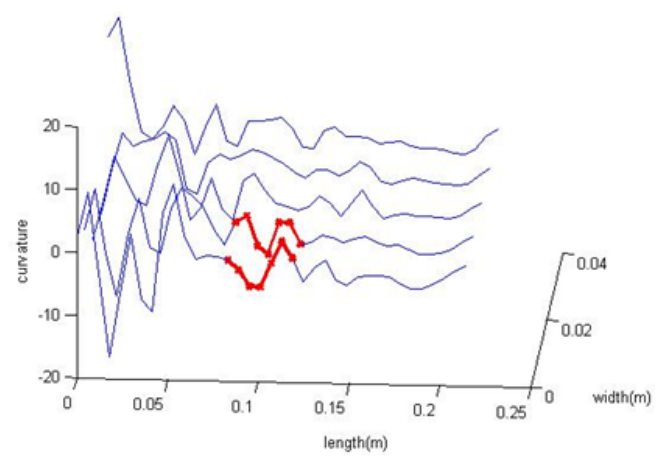

b)

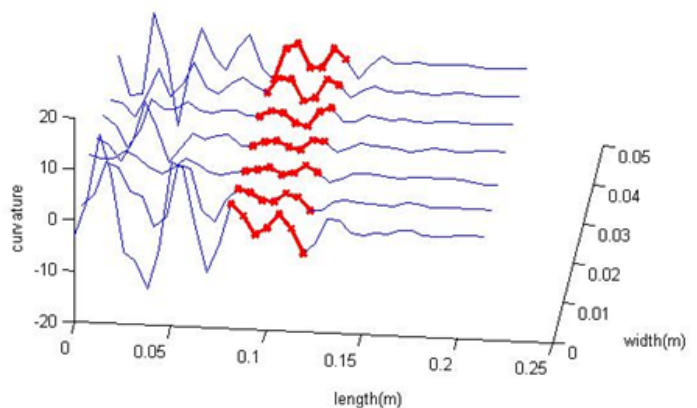

length(m)
c)

Figure 4.7: a) Healthy specimen b) C-type delamination c) Full width delamination

\subsubsection{Generalized fractal dimension technique}

Generalized fractal dimension technique is another widely used approach which does not require healthy structure information for damage location. Fractal dimension technique was proposed by Hadjileontiadis and Douka [35] and used for locating the crack in beams. But was not able to enhance the effect of delamination for higher mode. Later it was modified by Qiao et al [34] and applied to locate delamination in composite plates. Delamination in the structure will affect the local stiffness of the structure, which in turn will introduce small changes in their mode shapes. Evaluating fractal dimension on mode shapes data will enhance the small localized change and will help in detecting and locating the delamination. The expression for evaluating the generalized fractal dimension is given by

$$
\begin{gathered}
G F D=\frac{\log _{10} n}{\log _{10}\left(\frac{d_{s}}{l_{s}}\right)+\log _{10}(n)} \\
d_{s}=\sqrt{\left(y_{i+k}-y_{i}\right)^{2}+S^{2}\left(x_{i+k}-x_{i}\right)^{2}} \\
l_{s}=\sum_{j=1}^{M} \sqrt{\left(y_{i+j}-y_{i}\right)^{2}+S^{2}\left(x_{i+j}-x_{i}\right)^{2}}
\end{gathered}
$$

where $n$ is the number of nodes used in a single window, $x$ and $y$ are the system coordinates and $S$ is the scale factor which needs to be chosen carefully in order to detect the delamination successfully. 
For example, 2nd mode shape of full width delamination shows the delaminated region when the scaling factor is 133 , therefore guessing the scaling factor is difficult. In equation $4.5 d s$ is the maximum distance in the curve and $l s$ is the total length of the curve, $k$ is the size of the window. Qiao et al .[34] did not give a proper explanation on choosing the scaling factor $S$ for various mode shapes. In this work, the scaling factor was removed to compute the fractal dimension and a modified expression is given by,

$$
\begin{gathered}
M G F D=\frac{\log _{10} n}{\log _{10}\left(\frac{D_{s}}{L_{s}}\right)+\log _{10}(n)} \\
D_{s}=\sqrt{\left(\triangle y_{i+k}-\triangle y_{i}\right)^{2}+\left(\triangle x_{i+k}-\triangle x_{i}\right)^{2}} \\
l_{s}=\sum_{j=1}^{M} \sqrt{\left(\triangle y_{i+j}-\triangle y_{i}\right)^{2}+\left(\triangle x_{i+j}-\triangle x_{i}\right)^{2}}
\end{gathered}
$$

where in equation $4.8, \triangle x_{i}$ is the normalized nodal displacement with respect to maximum nodal displacement in xdirection, $\triangle y_{i}$ is the normalized nodal displacement with respective to maximum nodal displacements in ydirection. $M$ is the number of nodes present in the single window. $D_{s}$ is the difference in the normalized nodal displacement between the first and last node of the chosen window, $k$ is size of the window, $x$ is the out of plane deformation and $y$ is vertical distance of the node. To locate the delamination and compute the size of the delamination, GFD and MGFD were applied to mode shapes acquired from Ansys modeling. Firstly, through width delamination case taken for the study and the details of the specimen are shown in the Fig 4.8(a). GFD and MGFD were applied to the first mode shape of the composite beam and the results are shown in Fig 4.8b and Fig 4.8c respectively. Both the approaches are effectively capturing the delamination location and size as shown in the Fig. 4.8 Subsequently, we applied both the approaches to study

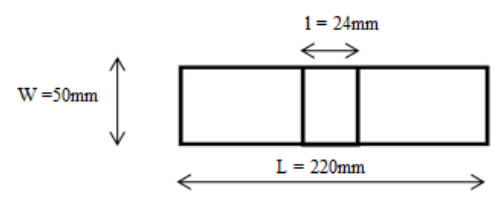

(a)

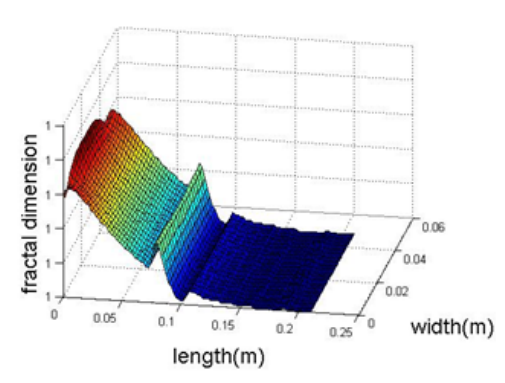

(b)

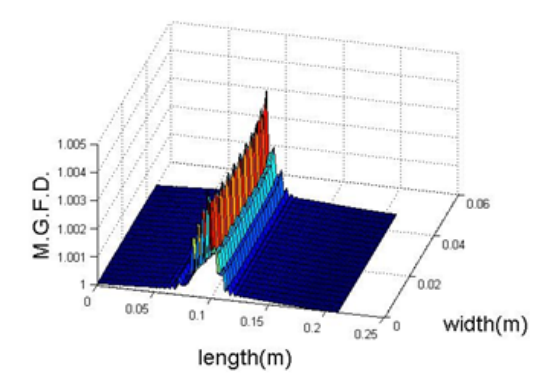

(c)

Figure 4.8: (a) Schematic diagram of beam having delamination at the center. (b) First mode by using fractal dimension. (c) First mode by using modified Fractal dimension Technique.

the delamination at the tip of the beam. The results of GFD and MGFD are presented in Fig. 4.9. GFD approach is not able to identify the delamination location at the tip of the beam, whereas MGFD can capture the delamination location and size more accurately. 


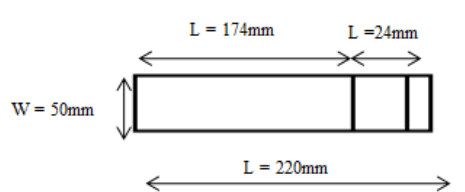

(a)

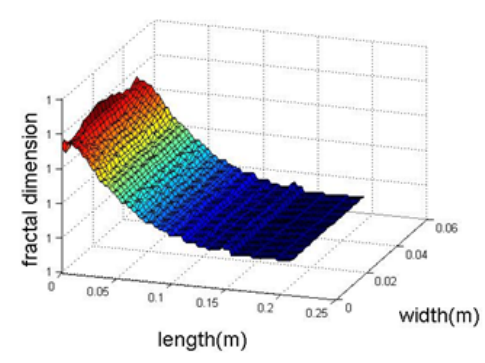

(b)

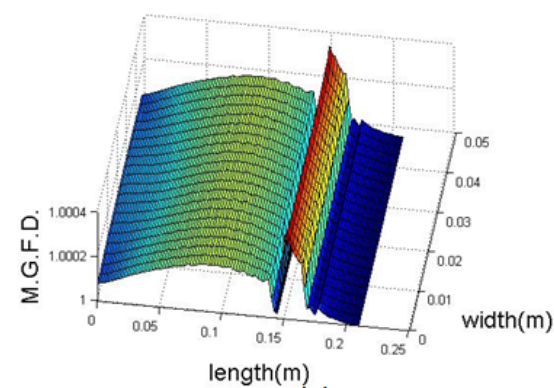

(c)

Figure 4.9: (a) Schematic diagram of beam having delamination at the tip. (b) First mode by using fractal dimension. (c) First mode by using Modified Fractal Dimension Technique.

Next, we investigated the minimum size of the delamination that can be identified by both the approaches. Fig. 4.10 shows that MGFD can capture delamination of size 2.2mm, whereas GFD fails to locate it. GFD and MGFD were then applied to study the multiple delamination case and the

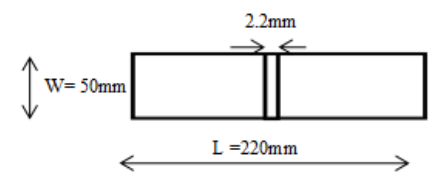

(a)

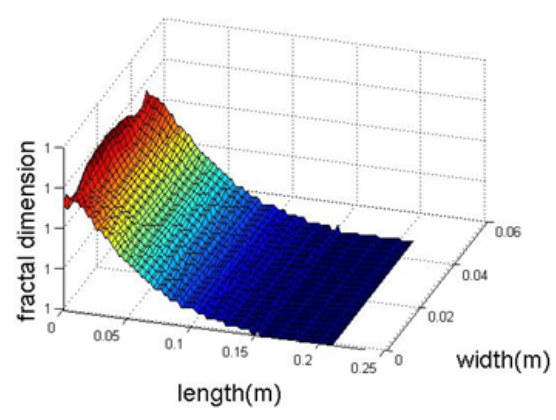

(b)

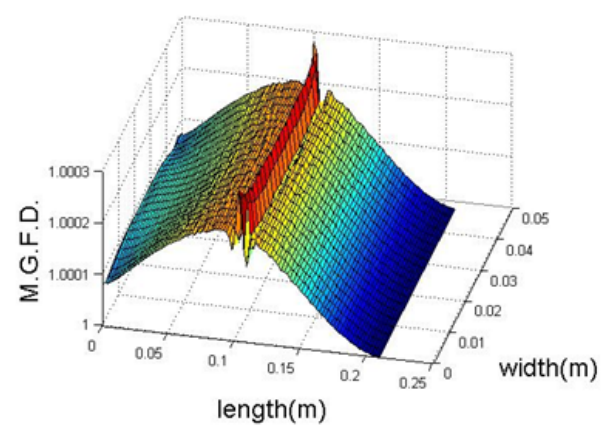

(c)

Figure 4.10: (a) Schematic diagram of beam having delamination at the center. (b) First mode by using fractal dimension. (c) First mode by using Modified Fractal Dimension Technique.

results are presented for the first mode shape in Fig. 4.11. GFD was able to locate the delamination near the fixed end, but was not able to capture the delamination at the tip Fig. 4.11c . MGFD was able to capture the delamination near the fixed end and the tip of the beam. Both the approaches were then applied to the fourth mode shape and the results are shown in Fig. 4.12. The results 


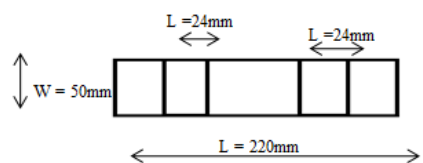

(a)

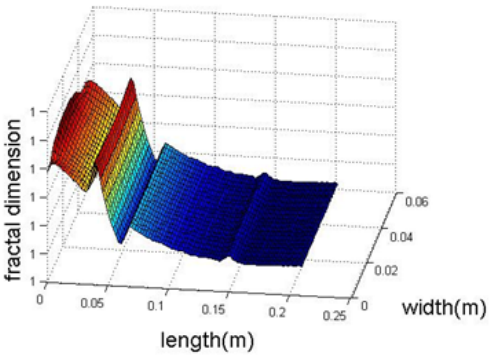

(b)

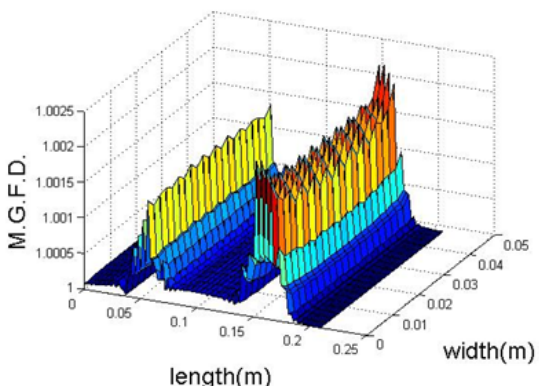

(c)

Figure 4.11: (a) Schematic diagram of beam having multiple delamination. (b) First mode by using fractal dimension. (c) First mode by using Modified Fractal Dimension Technique.

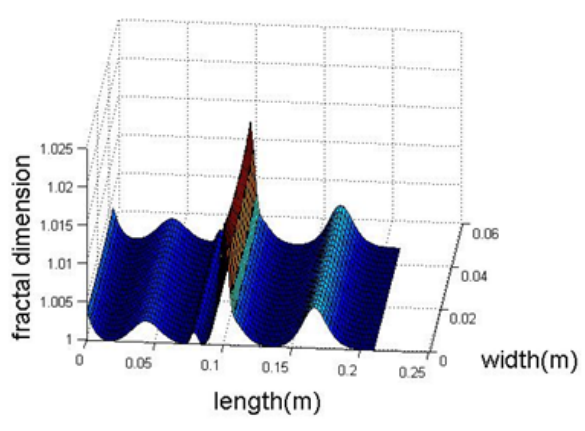

(a)

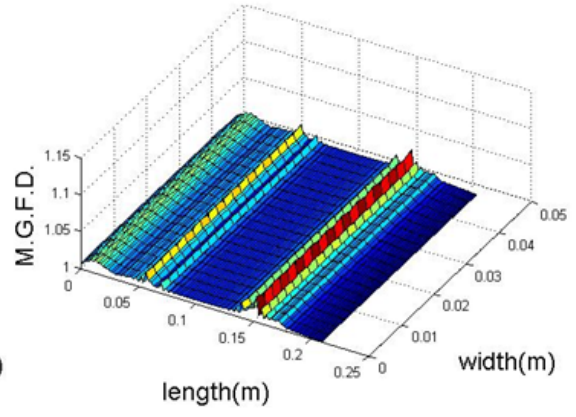

(b)

Figure 4.12: (a) Fourth mode by using fractal dimension. (b) Fourth mode by using Modified Fractal Dimension Technique.

in this section indicates the limitations of GFD for damage location and are summarized as follows 1) unable to detect the delamination present near the tip i.e. at the free end if it is cantilever, 2) when applied to higher modes gives wrong information regarding the delamination, 3) not able to locate delamination below a certain size, 4) proper selection of the scaling factor is important to get correct location of delamination. MGFD overcomes all the above limitations of GFD and the results in this section shows that it can capture location and size of delamination more accurately. Thus, the proposed MGFD was then applied to study the experimental mode shapes obtained by LDV in order to identify the delamination.

\section{Experimental results for GFRP composite specimen}

Experimental study was carried out on the unidirectional GFRP laminate $[0]_{4}$ specimen having dimension of $220 \mathrm{~mm} \times 50 \mathrm{~mm} \times 1.16 \mathrm{~mm}$. The material properties of GFRP laminate were obtained by ASTM standards tests and are given by $E_{11}=42.1 \mathrm{GPa}, E_{22}=10.3 \mathrm{GPa}, E_{33}=10.3 \mathrm{GPa}$, 
$G_{12}=3.84 \mathrm{GPa}, G_{23}=3.97 \mathrm{GPa}, G_{31}=3.84 \mathrm{GPa},{ }_{12}=0.27,{ }_{23}=0.37,{ }_{31}=0.27$ and density $=$ $2100 \mathrm{~kg} / \mathrm{m} 3$. The C-type and I-type delamination composite specimens were fabricated as per the information given in the reference .[?,30]. Experiments were carried out on the various delaminated specimen using LDV and the natural frequencies obtained are given in the table 4.1.The results in the table shows that both the experimental and finite element modeling results of the delaminated GFRP specimen are close to each other. From the frequency table, we are able to identify the presence of the delamination in the specimen. But we are not able to get exact location and size of the delaminated region. Subsequently MGFD were applied to the Numerical output of Healthy specimen and experimental output of healthy specimen as shown in fig 4.13. To validate the modification of GFD, MGFD were applied for the full width delamination which is working well as shown in fig. 4.14. Similarly, we fabricated the partial delamination namely I-type and Ctype with a reference of Kumar[29, 30] and applied MGFD, from fig. 4.15 and fig. 4.16 it is clearly visible to locate the length and width of delaminated portion. Further extension of work were carried out on the multiple delamination where more than one full width delamination is present and MGFD were successfully applied to numerical and experimental results fig. 4.17. After the

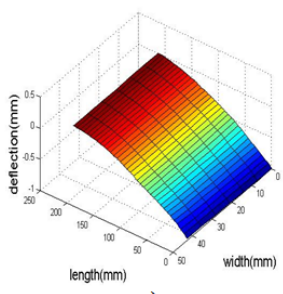

a)

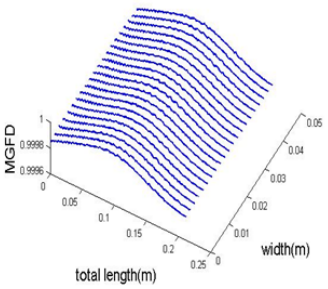

b)

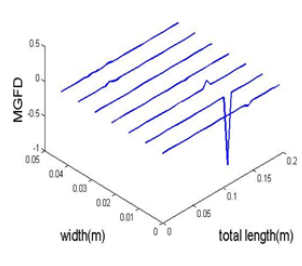

c)

Figure 4.13: (a) Mode shape of Healthy specimen mode shape b) MGFD on Numerical result c) MGFD on Experimental results

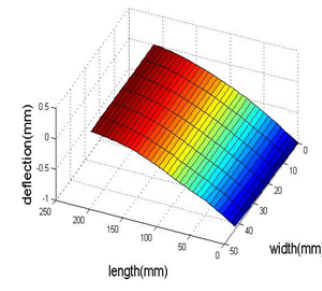

a)

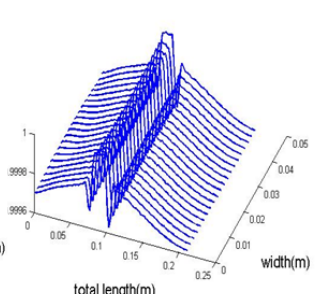

b)

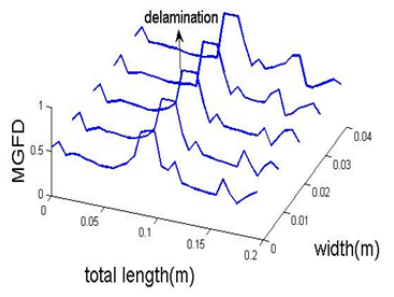

c)

Figure 4.14: (a) Mode shape of full width delaminated specimen b) MGFD on Numerical result c) MGFD on Experimental results 


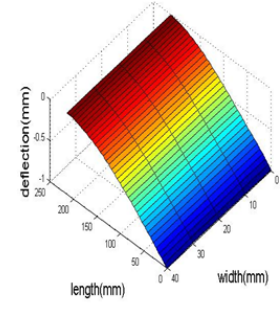

a)

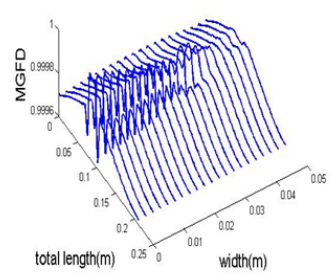

b)

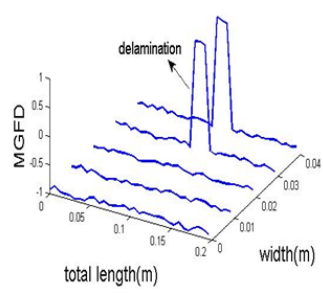

Figure 4.15: a) Mode shape of C-type delaminated specimen b) MGFD on Numerical result c) MGFD on Experimental results

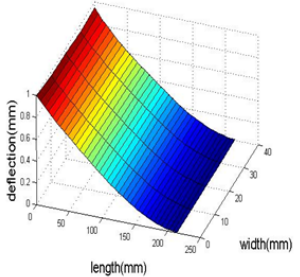

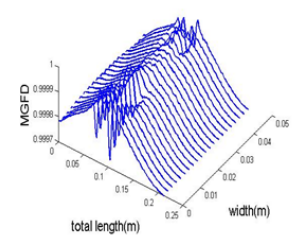

b)

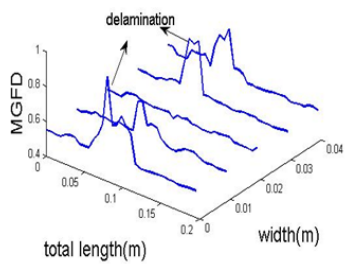

c)

Figure 4.16: a) Mode shape of I-type delaminated specimen b) MGFD on Numerical result c) MGFD on Experimental results

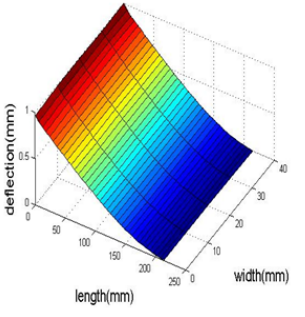

a)

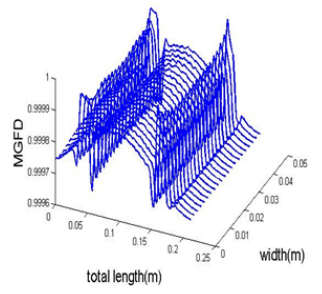

b)

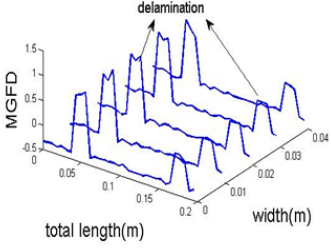

c)

Figure 4.17: a) Mode shape of Multiple delaminated specimen b) MGFD on Numerical result c) MGFD on Experimental results

successfully analysis of the beam, GFRP plate with dimensions $300 \mathrm{~mm} \times 150 \mathrm{~mm}$ was fabricated and were studied. Composite plate having $[0]_{4}$ with delamination present at the center and another plate having delamination at the edge and partial delamination near to fixed end. Fig. 4.18 and fig. 4.19 represents the schematic diagram and the MGFD plot of both the specimen. MGFD results were able to locate the single and multiple delamination accurately in the UD composite plates.

The modified fractal dimension technique was successfully applied to different kinds of partially delaminated specimen and were successfully able to locate and predict the size of the delamination in the lengthwise and widthwise directions. 
Table 4.1: Experimental and numerical results of various delaminated GFRP specimen.

\begin{tabular}{lllllllllll}
\hline $\begin{array}{l}\text { Mode } \\
\text { No. }\end{array}$ & \multicolumn{2}{l}{$\begin{array}{l}\text { Healthy } \\
\text { specimen }(\mathrm{Hz})\end{array}$} & \multicolumn{2}{l}{$\begin{array}{l}\text { Full-width } \\
\text { delamination }(\mathrm{Hz})\end{array}$} & \multicolumn{2}{l}{$\begin{array}{l}\text { C-type } \\
\text { delamination }(\mathrm{Hz})\end{array}$} & \multicolumn{2}{l}{$\begin{array}{l}\text { I-type } \\
\text { delamination }(\mathrm{Hz})\end{array}$} & $\begin{array}{l}\text { Multiple } \\
\text { delamination }(\mathrm{Hz})\end{array}$ \\
\hline & FEA & Exp & FEA & Exp & FEA & Exp & FEA & Exp & FEA & Exp \\
\hline First & 16.84 & 17.18 & 16.82 & 16.60 & 16.83 & 16.60 & 16.83 & 16.06 & 16.79 & 15.62 \\
\hline Second & 105.47 & 107.81 & 105.43 & 105.46 & 105.45 & 105.46 & 105.45 & 105.40 & 102.96 & 104.50 \\
\hline Third & 295.37 & 287.57 & 284.96 & 279.06 & 291.58 & 283.06 & 291.68 & - & 291.62 & 270.50 \\
\hline Fourth & 578.78 & 582.50 & 573.74 & 580.89 & 577.92 & 581.08 & 577.84 & - & 564.31 & 577.80 \\
\hline
\end{tabular}

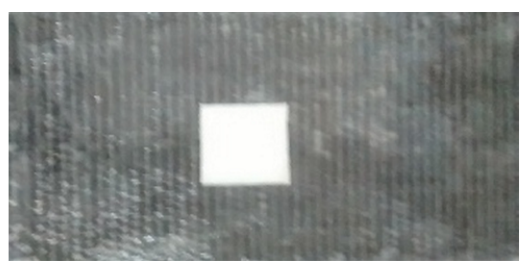

a)

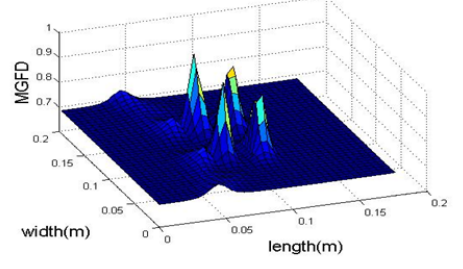

b)

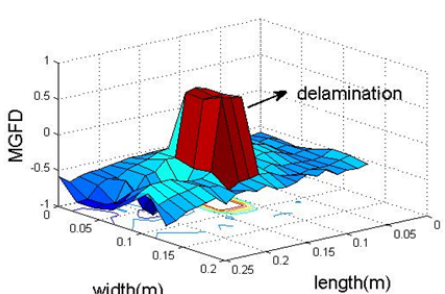

c)

Figure 4.18: a) schematic diagram of plate b) MGFD on Numerical result c) MGFD on Experimental results

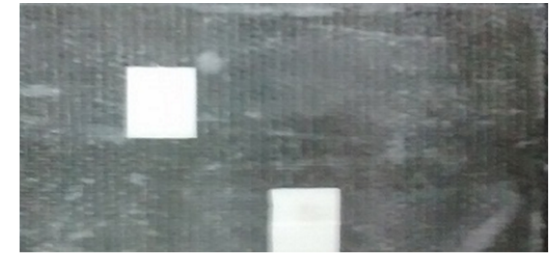

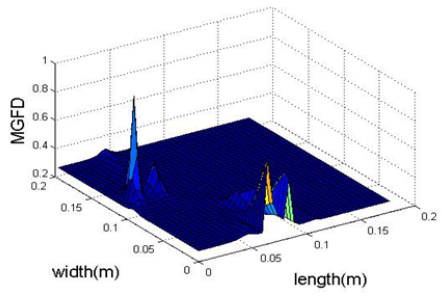

b)

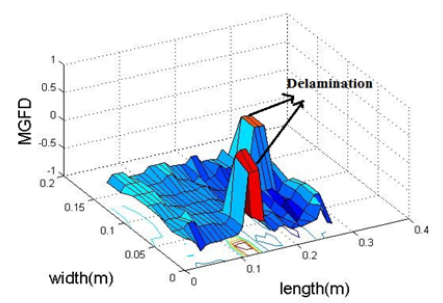

c)

Figure 4.19: a) schematic diagram of plate b) MGFD on Numerical result c) MGFD on Experimental results

\subsection{Summary}

Damage detection and quantification methods are essential for design of a robust SHM system. In this work, a modified generalized fractal dimension technique was applied to study the experimentally 
obtained mode shapes using LDV of partial and multiple delamination. MGFD was able to locate and quantify the size of the delamination in composite beam and plate structures. MGFD does not require any baseline information for location of the damage. Experimental and numerical results using MGFD can be used for damage quantification in composite structures. 


\section{Chapter 5}

\section{Conclusion and Recommendation for future work}

\subsection{Conclusions}

The thesis work focused on the identification, location and quantification of various partial delamination in the composite structures by performing the modal analysis using numerical and experimental work.

\subsubsection{Numerical work}

Finite element modeling procedures using commercial FEA package Ansys 14 were developed to simulate the various kinds of partial delamination like C-type and I-type using 3D brick and 2D contact finite elements. Modal analysis were then carried out to study the effect of delamination on the natural frequency and mode shapes. The FE results validated the available literature results.

\subsubsection{Experimental work}

Initially experimental modal analysis was performed using LDV for GFRP composite material characterization. Modal parameters like natural frequency and mode shapes of free-free supported GFRP plate was used to determine the elastic properties. Algorithms like RDFS was used to smooth the experimental acquired mode shapes and were then used in an inverse algorithm proposed by Grediac to determine the bending stiffness coefficients. Elastic properties of GFRP laminate determined from the modal analysis closely matched the results of DIC from static destructive tests. The obtained elastic properties of GFRP were then used in FE analysis for performing modal analysis of composite structures. Subsequently, the experimental program consist of fabrication of the E-glass/epoxy composite laminated beam with various type of delamination. The modal analysis test is conducted on healthy and damage specimens using laser Doppler vibrometer, and the responses are acquired in the form of modal parameters, i.e., natural frequencies and mode shapes. The natural frequency results obtained using LDV correlated well with the numerical FE analysis for all the partial delaminated composite beam structures. Subsequently, the mode shape acquired using LDV was studied using modified generalized fractal dimension technique to locate and quantify the size of the various 
delamination in the GFRP specimen. MGFD is a baseline free technique and was quite good in capturing the location and size of the delamination when compared to other methods like gapped smoothing method. MGFD technique was successfully extended to GFRP plates to detect and quantify single and multiple delaminations.

\subsection{Future work}

In the future, we want to extend our modal analysis approach using LDV to solve the inverse problem of locating the delamination in the thickness direction of the laminate. Further, we want to extend the MGFD approach to locate delamination in complex structures like stiffened composite

panels. From the numerical point of view, we want to develop continuum shell elements to model the different kinds of partial delamination for reducing the computational effort. 


\section{References}

[1] D.L. Balageas (2002) Structural health monitoring at the European Research Establishments in Aeronautics (EREA), Aerospace Science and Technology, 6, 159-170.

[2] J. Wu, S. Yuan, Y. Shang and Z. Wang (2009) Strain distribution monitoring wireless sensor network design and its evaluation research on aircraft wing-box, International Journal of Applied Electromagnetic and Mechanics, 31, 17-28.

[3] Ch.E. Katsikeros and G.N. Labeasa (2009) Development and validation of a strain-based Structural Health Monitoring system, Mechanical Systems and Signal Processing, 23(2), 372-383.

[4] X. Qing, A. Kumar, C. Zhang, I.F. Gonzalez, G. Guo and F.K. Chang (2005) A hybrid Piezoelectric/Fiber optic diagnostic system for structural health monitoring, Smart Materials and Structures, 14, 98-103.

[5] V. Giurgiutiu and C. A. Rogers (1997) Electro-Mechanical Impedance Method for Structural Health Monitoring an Non-Destructive Evaluation, Int. Workshop on Structural Health Monitoring, Stanford University, California, Sep. 18-20, 433-444.

[6] V. Giurgiutiu, A. N. Zagrai (2000) Damage Detection in Simulated Aging-Aircraft Panels Using the Electro-Mechanical Impedance Technique, Adaptive Structures and Materials Systems Symposium, ASME Winter Annual Meeting, November 5-10, Orlando, Florida.

[7] G. Park, H. Sohn, C. Farrar and D. Inman 2003, Overview of Piezoelectric Impedance-Based Health Monitoring and Path Forward, Shock and Vibration Digest, 35(6), 451-463.amaged location and the transducer.

[8] G. Aranguren,P.M Monje, Valerijan Cokonaj, Edurdo Barrera and Mariano Ruiz, "Ultrasonic wave-based structural health monitoring embedded instrument." REVIEW OF SCIENTIFIC INSTRUMENTS 84, 125106 (2013)

[9] S. W. Doebling, C. R. Farrar, M. B. Prime, and D. W. Shevitz (1996) Damage identification and health monitoring of structural and mechanical systems from changes in their vibration characteristics : A literature review, Technical Memorandum LA-13070-MS, Los Alamos National Laboratory.

[10] Y.Zou, L. Tong and G.P. Steven(1998) Vibration-based model- dependent damage (delamination) identification and health monitoring for composite structures-A review it Journal of Sound and Vibration (200), bf 230(2), 357-378. 
[11] Obinna K. Ihesiulor *, Krishna Shankar, Zhifang Zhang, Tapabrata Ray, Delamination detection with error and noise polluted natural frequencies using computational intelligence concepts, Composites: Part B 56 (2014) 906-925.

[12] Ramkumar, R.L., S.V., and pipes, R.B.1979 Free vibration frequencies of a delamination Beam 34th Annual Technical Conference Proceeding, Reinforced/Composite Institute, Society of Plastic Industries, Section 22-E,I-5

[13] Wang, J.T.S., Liu, Y.Y., and Gibby , J.A.,1982," Vibration of split Beams." J.sound Vib., 84, pp.491-502

[14] Mujumdar P, Suryanarayan S. Flexural vibration of the beams with delamination. J sound Vib 1988;125(3):441-61

[15] Chakraborty D. Artifical neural network based delamination prediction in laminated composites. Mater Des 2005;26:17

[16] Adams RD, Cawley P. A vibration technique for non-destructive assessing the integrity of the structures. J. Mech Eng Sci 1978;20(2): 93-100

[17] C. N. Della and D. Shu. Vibration of delaminated composite laminates: A review. Applied Mechanics Reviews, 60(1):120, 2007.

[18] Nagesh Babu, G.L., and Hanagud, S.,1990," Delamination in smart structure, A parametric Study on Vibration ," 31st AIAA/ASME/ASCE/AHS/ASC structures, Structural Dynamics and Material Conference, Long Beach,CA, April 2-4,pp.2417-2426

[19] Krawczuk, M., Ostachowicz, W., and zak,A.,1996, "Analysis of natural frequencies of delamination composite beams based on finite element method ", Struct. Eng. Mech.,4,pp.243-255.

[20] Krawczuk, M., Zak, A., and ostachowicz, W., 1997, "Dynamics of cracked composite material structures," Comput, Mech.,20,,pp. 79-83

[21] Zak, A., Krawczuk, M., and ostachowicz, W., 2000, "Numerical and Experimental Investigation of free vibration of Multilayer Delaminated Composite Beams and Plates," Comput. Mech.,26,pp.309-315

[22] Ju,F., Lee, H.P.,and Lee, K.H.,1994,"Free-Vibration Analysis of Composite Beams with multiple delamination," Composites Eng., 4,pp. 715-730

[23] Campanelli, R, W., and Engblom, J. J., 1995, " The effect of delamination in Graphit/PEEK Composite Plates on Modal Dynamic Characteristics," Compos. Struct., 31,pp. 195-202

[24] Okafor AC, Chandrashekar K, Jiang YP. Delamination prediction in composite beams with built-in piezoelectric devices using modal analysis and neural network. Smart Mater Struct $1996 ; 5 ; 338-47$.

[25] Valoor MT, Chandrashekhara K. A thick composite-beam model for delamination prediction by the use of neural networks. Compos Sci Technoll2000;60:1773-9 
[26] Zhifang Zhang,Krishna Shankar, Tapabrata Ray, Egeny V. Morozov, Murat Tahtali, "Vibrationbased inverse algorithms for detection of delamination in composite" J. of Composite structure 102(2013) 226-236

[27] M.H. Shen and J.Grady. Free vibration of delaminated beams. AIAA journal 30,(1992)1310-1370

[28] S. Keshav Kumar. Delamination modelling and detection in composite structure P.H.D. thesis, Indian Institute of Science, Bangalore.

[29] S. Keshav Kumar. Delamination modelling and detection in composite structure P.H.D. thesis, Indian Institute of Science, Bangalore.

[30] S.keshav kumar ,Ranjan Ganguli, Dinesh kumar Harursampath, Partial delamination modelling in composite beams using a finite elemnt method, Finite element in analysis and design volume 75 pages $1-12$

[31] Lestari W, P.Qia, and S.Hanagud. Curvature mode shape based damage assessment of carbon/epoxy composite beam. Journal of intelligent material system and structure 18,2007 189208.

[32] S.S. Kessler, S.M. Spearing, M.J. Atalla, C.E. cesnik, and C.Soutis. Damage detection in composite material using frequency response method. composite partB Engineering 33,(2002)87-95

[33] Mark N. Helfrick, Christopher Niezrecki, Peter Avitabile, Timothy Schmidt,"3D digital image correlation methods for full-field vibration measurement",J. of Mechanical Systems and Signal Processing 25(2011) 917-927.

[34] Pizhong Qiao, *, Kan Luc, Wahyu Lestarid, Jialai Wange, Curvature mode shape-based damage detection in composite laminated plates, Composite Structures 80 (2007) 409428.

[35] L.J. Hadjileontiadisa, ${ }^{*}$, E. Doukab, A. Trochidisc Fractal dimension analysis for crack identification in Beam structures Mechanical Systems and Signal Processing 19 (2005) 659674

[36] P.Cawley and R.Adams. The location of defects in structures from measurement of natural frequencies. The journal of strain analysis for Engineering Design 14,(1979) 49-57

[37] D.F.Adams,L.A.Carlsson,R.B.Pipes, Experimental Characterization of Advanced Composite Materials, CRCPress,BocaRaton, 2003.

[38] M.Grediac AND P. A.Paris Direct Identification of elastic constant of anisotropic plates by modal analysis: Therotical and numerical aspect.Journal of Sound and Vibration (1996) 195(3), 401-415

[39] M.Grediac and A Vautrin 0882 European Journal of Mechanics A:Solids 01708727 Mechanical characterization of anisotropic plates experiments and results.

[40] T.H Ooijevaar. Vibration based structural health monitoring of composite skin-stiffener structures. Universities Twente, 2014 
[41] F.B. Batista, E.L.Albuquerque, J.R.F.Arruda, M.DiasJr. Identification of the bending stiffness matrix of symmetric laminates using regressive discrete Fourier series and finite differences.Journal of Sound and Vibration 320 (2009) 793807

[42] Wei Fan and Pizhong Qiao Vibration-based Damage Identification Methods: a review and Comparative study, J. Structral Health Monitoring Vol 10(1);83-29

[43] WeiFan and Pizhong Qiao, Vibration based Damage Identification methods A review and comparative study, Structral Health monitoring vol10(1) 83-29.

[44] M. H. H. Shen and J. E. Grady. Free vibrations of delaminated beams. AIAA Journal, 30(5) :1361

[45] C.R Farrar and S.W. Doebling. An overview of modal-based damage identification methods. In proceedings of DAMAS conference. Citeseer, 1997 269-278.

[46] J.R.F.Arruda, Surface smoothing and partial spatial derivatives computation using aregressive discrete Fourier series, Mechanical Systems andSignalProcessing 6 (1992)4150.

[47] Sohn, H., Farrar, C.,Hunter, N. and Worden, K.(2003). A review of structural Health Monitoring Literature: 1996-2001. Los Alamos National Laboratory report, (LA-13976-MS)

[48] Gudmundson, P(1982). Eigen frequency changes of structure due to cracks, notches or other geometrical changes, Journal of the Mechanics and Physics of solids, 30,339-353

[49] Liang, R.Y., Hu, J.L. and choy, F.(1992). Therotical-study of crack-induced eigenfrequency changes on beam structures. Journal of engineering Mechanics-ASCE, 118,384-396

[50] Pandey, A.K., Biswas, M. and Samman, M.M.(1991) Damage detection from change in curvature mode shapes. Journal of sound and vibration, 145,321-332

[51] M. Rucka, , K. Wilde Application of continuous wavelet transform in vibration based damage detection method for beams and plates. J. Sound Vib. 297, 536-550

[52] Shuncong ZhongS. Olutunde, Oyadiji S. Olutunde Oyadiji, Crack detection in simply supported beams without baseline modal parameters by stationary wavelet transform Mechanical Systems and Signal Processing 21(4):1853-1884 May 2007 